\title{
Binary Asteroid Systems: Tidal End States and Estimates of Material Properties
}

\author{
Patrick A. Taylor ${ }^{1}$ and Jean-Luc Margot ${ }^{2}$ \\ ${ }^{1}$ Arecibo Observatory, ${ }^{2} \mathrm{UCLA}$ \\ ptaylor@naic.edu
}

Published in Icarus

Submitted 29 June 2010

Revised 17 January 2011

Accepted 18 January 2011

Available Online 1 February 2011

Published in Print April 2011 in Volume 212, pp. 661-676

Manuscript Pages: 39

Tables: 3

Figures: 6

NOTICE: this is the author's version of a work that was accepted for publication in Icarus. Changes resulting from the publishing process, such as peer review, editing, corrections, structural formatting, and other quality control mechanisms may not be reflected in this document. Changes may have been made to this work since it was submitted for publication. A definitive version was subsequently published in Icarus 212, 661-676, April 2011, DOI: 10.1016/j.icarus.2011.01.030.

Publisher's copy: http://dx.doi.org/10.1016/j.icarus.2011.01.030 


\section{ABSTRACT:}

The locations of the fully despun, double synchronous end states of tidal evolution, where the rotation rates of both the primary and secondary components in a binary system synchronize with the mean motion about the center of mass, are derived for spherical components. For a given amount of scaled angular momentum $J / J^{\prime}$, the tidal end states are over-plotted on a tidal evolution diagram in terms of mass ratio of the system and the component separation (semimajor axis in units of primary radii). Fully synchronous orbits may not exist for every combination of mass ratio and angular momentum; for example, equal-mass binary systems require $J / J^{\prime}>0.44$. When fully synchronous orbits exist for prograde systems, tidal evolution naturally expands the orbit to the stable outer synchronous solution. The location of the unstable inner synchronous orbit is typically within two primary radii and often within the radius of the primary itself. With the exception of nearly equal-mass binaries, binary asteroid systems are in the midst of lengthy tidal evolutions, far from their fully synchronous tidal end states. Of those systems with unequal-mass components, few have even reached the stability limit that splits the fully synchronous orbit curves into unstable inner and stable outer solutions.

Calculations of material strength based on limiting the tidal evolution time to the age of the Solar System indicate that binary asteroids in the main belt with 100-km-scale primary components are consistent with being made of monolithic or fractured rock as expected for binaries likely formed from sub-catastrophic impacts in the early Solar System. To tidally evolve in their dynamical lifetime, near-Earth binaries with km-scale primaries or smaller created via a spin-up mechanism must be much weaker mechanically than their main-belt counterparts even if formed in the main belt prior to injection into the near-Earth region. Small main-belt binaries, those having primary components less than $10 \mathrm{~km}$ in diameter, could bridge the gap between the large main-belt binaries and the near-Earth binaries, as, depending on the age of the systems, small main-belt binaries could either be as strong as the large main-belt binaries or as weak as the near-Earth binaries. The inherent uncertainty in the age of a binary system is the leading source of error in calculation of material properties, capable of affecting the product of rigidity $\mu$ and tidal dissipation function $Q$ by orders of magnitude. Several other issues affecting the calculation of $\mu Q$ are considered, though these typically affect the calculation by no more than a factor of two. We also find indirect evidence within all three groups of binary asteroids that the semimajor axis of the mutual orbit in a binary system may evolve via another mechanism (or mechanisms) in addition to tides with the binary YORP effect being a likely candidate.

Keywords: Asteroids - Satellites of Asteroids - Tides, solid body - Asteroids, dynamics Near-Earth objects 


\section{Introduction}

Over 20 years ago, Weidenschilling et al. (1989) asked, "Do asteroids have satellites?" Today, binary systems have been discovered in every dynamical class of small Solar System bodies from near-Earth asteroids to Mars-crossers and main-belt asteroids, among the Jupiter Trojans, and in the outer Solar System in the Kuiper belt. Beginning with the Galileo spacecraft's serendipitous discovery in 1993 of tiny satellite Dactyl orbiting (243) Ida (Chapman et al., 1995; Belton et al., 1996) while on its cruise to Jupiter and continuing with the success of radar, lightcurve photometry, ground-based adaptive-optics imaging, and Hubble Space Telescope imaging, reviewed by Merline et al. (2002), Richardson and Walsh (2006), and Noll et al. (2008), over 180 small Solar System bodies are suspected to be binary or multiple systems 1 In this work, we build upon the ideas presented in Weidenschilling et al. (1989) to illustrate the tidal end states of binary asteroid systems and discuss the inherent material strength of the bodies implied by tidal evolution.

The Keplerian orbit of the two components in a binary system about the center of mass allows one to determine the mass of the system and, with an estimate of the component sizes, the (assumed equal) densities of the bodies. Density estimates combined with taxonomic classifications hint at the internal structure of asteroids (Britt et al., 2002). When compared to analog meteorites with known bulk densities, low densities found for rocky asteroids imply that, rather than monolithic bodies, asteroids are aggregate bodies made up of many fragments with varying degrees of porosity. Aggregates can range from low-porosity fractured or shattered bodies to porous, cohesionless rubble piles with "gravitational aggregate" acting as a catch-all term for bodies comprised of many fragments, independent of porosity, that are held together by their collective gravity (Richardson et al., 2002). The tidal evolution of these binary systems is intimately tied to the internal structure and material strength of the bodies involved, and we will exploit this dependence to estimate the combined effect of rigidity and energy dissipation in the bodies to determine if asteroids tend to have solid, fractured, or shattered interiors.

Hints of the internal structure of asteroids also come from the most probable binary formation mechanisms for each population: near-Earth binaries likely form through rotational disruption via spin-up by thermal torques due to sunlight (the YORP effect; Bottke et al., 2002; Walsh et al., 2008) or close planetary encounters (Richardson et al., 1998; Walsh and Richardson, 2006), 1- to 10-km-scale binaries in the main belt may also form through YORP spin-up (Pravec and Harris, 2007), 100-km-scale main-belt binaries likely form through sub-catastrophic impacts (e.g., Durda et al., 2004), and Kuiper-belt binaries may have formed very early on via gravitational collapse (Nesvorný et al., 2010) or later on via collisions or a flavor of dynamical capture (see Noll et al., 2008, for a review). Binary formation through rotational disruption at the very least implies a fractured interior, and if satellite formation is due to the accretion of smaller particles spun off the parent body, as modeled by Walsh et al. (2008), part or all of the parent body is

\footnotetext{
${ }^{1}$ The value of more than 180 suspected binary or multiple systems is taken from http://www.johnstonsarchive.net/astro/asteroidmoons.html and based on references therein. Lists of binary and multiple asteroid parameters are available from the Ondrejov Asteroid Photometry Project (http://www.asu.cas.cz/ asteroid/binastdata.htm) and the Planetary Data System (http://sbn.psi.edu/pds/asteroid/EAR_A_COMPIL_5_BINMP_V3_0/data/).
} 
likely an aggregate of smaller pieces and structurally weak. For sub-catastrophic impacts, the satellite is either a solid shard or an aggregate made up of impact ejecta while the parent body may remain solid or fractured rather than shattered. Thus, we anticipate that examination of tidal evolution in binary asteroid systems will find that large main-belt binaries are consistent with solid or fractured rock, while near-Earth and small main-belt binaries are consistent with structurally weaker, shattered or porous gravitational aggregates.

Section 2 introduces the important dynamical quantities of binary systems, angular momentum and energy. In Section 3, we derive and illustrate for a range of angular momenta the fully despun, double synchronous orbits that are end states of tidal evolution and then discuss stability limits and energy regimes in Section 4. Using the known properties of binary asteroids in the near-Earth region and the main belt, in Section 5 we constrain the material properties of the bodies based on tidal evolution.

\section{Angular momentum and energy content}

The dynamics of a binary system with primary of mass $M_{\mathrm{p}}$ and secondary of mass $M_{\mathrm{s}}$ in a mutual orbit about their common center of mass can be described equivalently by a system where a body of mass $M_{\mathrm{p}} M_{\mathrm{s}} /\left(M_{\mathrm{p}}+M_{\mathrm{s}}\right)$ orbits a stationary mass, $M_{\mathrm{p}}+M_{\mathrm{s}}$. The orbital angular momentum of such a system is $L=M_{\mathrm{p}} M_{\mathrm{s}} /\left(M_{\mathrm{p}}+M_{\mathrm{s}}\right) \sqrt{G\left(M_{\mathrm{p}}+M_{\mathrm{s}}\right) a\left(1-e^{2}\right)}$ where $G$ is the gravitational constant and $a$ and $e$ are the semimajor axis and eccentricity of the mutual orbit, respectively. Defining the component mass ratio $q=M_{\mathrm{s}} / M_{\mathrm{p}}=\left(\rho_{\mathrm{s}} / \rho_{\mathrm{p}}\right)\left(R_{\mathrm{s}} / R_{\mathrm{p}}\right)^{3} \leq 1$, where $\rho$ and $R$ are the bulk density and radius, respectively, of the components, using the system mass $M_{\text {sys }}=M_{\mathrm{p}}+M_{\mathrm{s}}=(1+q) M_{\mathrm{p}}$, and applying Kepler's Third Law, $n^{2} a^{3}=G\left(M_{\mathrm{p}}+M_{\mathrm{s}}\right)$ in terms of the mean motion $n$, the orbital angular momentum is:

$$
L=\frac{q}{(1+q)^{2}} M_{\mathrm{sys}} a^{2} n\left(1-e^{2}\right)^{1 / 2}=\frac{q}{1+q} M_{\mathrm{p}} a^{2} n\left(1-e^{2}\right)^{1 / 2} .
$$

For principal axis rotation, the spin angular momentum of the system is given by the moments of inertia $I=\alpha M R^{2}$ of the components and their spin rates $\omega$ as:

$$
S=I_{\mathrm{p}} \omega_{\mathrm{p}}+I_{\mathrm{s}} \omega_{\mathrm{s}}=\alpha_{\mathrm{p}} M_{\mathrm{p}} R_{\mathrm{p}}^{2} \omega_{\mathrm{p}}\left(1+\frac{\alpha_{\mathrm{s}}}{\alpha_{\mathrm{p}}}\left(\frac{\rho_{\mathrm{p}}}{\rho_{\mathrm{s}}}\right)^{2 / 3} q^{5 / 3} \frac{\omega_{\mathrm{s}}}{\omega_{\mathrm{p}}}\right) .
$$

The coefficient $\alpha$ is $2 / 5$ for a uniform-density, rotating sphere, but varies with the shape of the body and the density profile of the interior.

For comparison, suppose a rapidly spinning, uniform-density parent body sheds mass in such a way to conserve angular momentum and produce the aforementioned mutually orbiting binary system. When a spherical body with mass $M$ and radius $R$ spins at the breakup rate $\omega_{\text {break }}$ without cohesion among its constituent particles, the inward acceleration due to gravity $G M / R^{2}$ at the equator is balanced by the outward centrifugal acceleration $\omega_{\text {break }}^{2} R$ due to rotation such that $\omega_{\text {break }}=\sqrt{G M / R^{3}}$. The angular momentum $J$ contained in the critically rotating sphere is $I \omega_{\text {break }}$ or:

$$
J=\frac{2}{5} \sqrt{G M_{\mathrm{sys}}^{3} R_{\mathrm{eff}}},
$$


where the mass and radius of the parent body have been written as the total mass and effective radius of the binary system, $R_{\mathrm{eff}}=\left(R_{\mathrm{p}}^{3}+R_{\mathrm{s}}^{3}\right)^{1 / 3}=\left(1+\frac{\rho_{\mathrm{p}}}{\rho_{\mathrm{s}}} q\right)^{1 / 3} R_{\mathrm{p}}$, produced by the breakup of the parent body. Subsequently, the total angular momentum of a binary system $J=L+S$ is often normalized by $J^{\prime}=\sqrt{G M_{\text {sys }}^{3} R_{\text {eff }}}$ (attributed to Darwin $(1887)$ ) such that $J / J^{\prime} \sim 0.4$ indicates the binary could have formed by mass shedding from the spin-up of a single spherical strengthless parent body. Pravec and Harris (2007) normalize the total angular momentum of the binary system by that of the critically rotating spherical parent body (Eq. 3) such that their scaling parameter $\alpha_{\mathrm{L}}=\left(J / J^{\prime}\right) / 0.4$, and $\alpha_{\mathrm{L}}=1$ has the same implication for binary formation. We utilize the $J^{\prime}$ normalization throughout this work.

The minimum and maximum separations of two components in a binary system are limited by the physical size of the components and the total angular momentum of the system, respectively. The separation of two components is naturally bounded from below by the contact condition where, at a separation of $R_{\mathrm{p}}+R_{\mathrm{s}}$, the components are resting against one another. In terms of the semimajor axis and the radius of the primary, the contact limit is:

$$
\left(\frac{a}{R_{\mathrm{p}}}\right)_{\text {min }}=1+\frac{R_{\mathrm{s}}}{R_{\mathrm{p}}}=1+\left(\frac{\rho_{\mathrm{p}}}{\rho_{\mathrm{s}}} q\right)^{1 / 3} .
$$

The contact limit ranges from $a / R_{\mathrm{p}}=1$ to 2 , akin to a pea resting on the surface of a basketball to two basketballs in contact. One could consider the Roche limit as a soft lower bound on the separation of the bodies, as opposed to the hard limit of physical contact, but with a modest amount of cohesion, a secondary may exist within the Roche limit and down to the contact limit without disrupting (Holsapple and Michel, 2008; Taylor and Margot, 2010). An upper bound is placed on the separation of the components by the total angular momentum content of the system. If the entire budget of angular momentum $J=\left(J / J^{\prime}\right) \sqrt{G M_{\mathrm{sys}}^{3} R_{\mathrm{eff}}}$ is transferred to the circular mutual orbit of the components, which are no longer spinning ( $S \rightarrow 0$ in Eq. 2), the maximum attainable separation according to Eq. 1 with $e=0$ is:

$$
\left(\frac{a}{R_{\mathrm{p}}}\right)_{\max }=\left(J / J^{\prime}\right)^{2} \frac{(1+q)^{4}}{q^{2}}\left(1+\frac{\rho_{\mathrm{p}}}{\rho_{\mathrm{s}}} q\right)^{1 / 3},
$$

which increases quickly with decreasing $q$, allowing for binaries with smaller secondaries to have much wider separations than binaries with similar-size components for the same amount of angular momentum.

Ignoring external influences on the system, the total energy of the binary $E$ is comprised of the rotations of the components $(1 / 2) I \omega^{2}$, the orbital motion about the center of mass $(1 / 2) M_{\mathrm{p}} M_{\mathrm{s}} /\left(M_{\mathrm{p}}+M_{\mathrm{s}}\right) a^{2} n^{2}$, and the mutual gravitational potential for a two-sphere system $-G M_{\mathrm{p}} M_{\mathrm{s}} / a$ such that:

$$
E=\frac{1}{2} I_{\mathrm{p}} \omega_{\mathrm{p}}^{2}+\frac{1}{2} I_{\mathrm{s}} \omega_{\mathrm{s}}^{2}-\frac{1}{2} \frac{M_{\mathrm{p}} M_{\mathrm{s}}}{M_{\mathrm{p}}+M_{\mathrm{s}}} a^{2} n^{2}
$$

While shape deformation may occur due to changes in spin rate (Harris et al., 2009; Holsapple, 2010) as the binary system evolves, we assume that this does not result in significant interchange of self-potential energy with the terms in Eq. 6. The energy accounted for in Eq. 6 is free to be 
traded among its components via the dynamical interaction of the two bodies, and the total energy can be dissipated as heat as a result of internal friction due to tidal flexure. Depending on the amount of rotational and translational energy of the system compared to the mutual gravitational potential energy, the total energy may either be positive or negative. A negative total energy requires the system to remain bound, while a positive total energy could allow the components to unbind themselves through their dynamical interaction.

\section{Fully synchronous orbits}

The natural evolution of a binary system is through mutual tidal interaction. The differential gravity across each body due to the proximity of its companion acts to evolve the rotation states of the individual bodies as well as their physical separation; tides tend to evolve rapidly rotating components in close proximity to a more widely separated system with more slowly rotating components. The end state of tidal evolution for a binary system is a fully despun, double synchronous orbit (hereafter called a fully synchronous orbit), where the spin rates of both components, $\omega_{\mathrm{p}}$ and $\omega_{\mathrm{s}}$, equal the mean motion in the mutual orbit $n$. The classic example of such an end state is in the Pluto-Charon system where the bodies are face-locked (Christy and Harrington, 1978), meaning they keep the same sides facing one another because the periods of rotation and revolution have synchronized.

The scaled angular momentum of the system $J / J^{\prime}$ at any time is given by the sum of Eq. 1 and Eq. 2 divided by $J^{\prime}$. Assuming a circular mutual orbit, which the majority of binary asteroid systems have, and after setting the spin rates $\omega_{\mathrm{p}}$ and $\omega_{\mathrm{s}}$ equal to the mean motion $n$, replacing $n$ with a function of the semimajor axis $a$ via Kepler's Third Law 2 and some rearranging, the locations of the fully synchronous orbits $a_{\mathrm{sync}} / R_{\mathrm{p}}$ are the solutions to the quasi-quadratic equation:

$$
\frac{1}{\alpha_{\mathrm{p}}} \frac{q}{1+q} \frac{1}{1+\frac{\alpha_{\mathrm{s}}}{\alpha_{\mathrm{p}}}\left(\frac{\rho_{\mathrm{p}}}{\rho_{\mathrm{s}}}\right)^{2 / 3} q^{5 / 3}}\left(\frac{a_{\mathrm{sync}}}{R_{\mathrm{p}}}\right)^{2}-\frac{J / J^{\prime}}{\alpha_{\mathrm{p}}} \frac{\left(1+\frac{\rho_{\mathrm{p}}}{\rho_{\mathrm{s}}} q\right)^{1 / 6}(1+q)}{1+\frac{\alpha_{\mathrm{s}}}{\alpha_{\mathrm{p}}}\left(\frac{\rho_{\mathrm{p}}}{\rho_{\mathrm{s}}}\right)^{2 / 3} q^{5 / 3}}\left(\frac{a_{\mathrm{sync}}}{R_{\mathrm{p}}}\right)^{3 / 2}+1=0 .
$$

If the components are spherical and have similar uniform densities, the condition for a fully synchronous orbit reduces to:

$$
\frac{5}{2} \frac{q}{1+q} \frac{1}{1+q^{5 / 3}}\left(\frac{a_{\mathrm{sync}}}{R_{\mathrm{p}}}\right)^{2}-\frac{5}{2} \frac{(1+q)^{7 / 6}}{1+q^{5 / 3}}\left(J / J^{\prime}\right)\left(\frac{a_{\mathrm{sync}}}{R_{\mathrm{p}}}\right)^{3 / 2}+1=0 .
$$

Depending on the total angular momentum of the system $J / J^{\prime}$ and the mass ratio $q$, the equation above may have two, one, or zero real solutions corresponding to inner and outer fully synchronous orbits, a single degenerate fully synchronous orbit, or the absence of a valid fully synchronous orbit, respectively, for the system. Solutions to Eq. 8 for all $q$ are shown in Fig. 1 for various values of $J / J^{\prime}$ where the number of solutions for a specific $q$ is given by the number of intersections between the curve of the relevant $J / J^{\prime}$ value for the system and the horizontal line of constant mass ratio

\footnotetext{
${ }^{2}$ In using $n^{2} a^{3}=G\left(M_{\mathrm{p}}+M_{\mathrm{s}}\right)$ here, we imply the components of the binary system have spherical shapes or may be treated as point masses.
} 
$q$ that the system will tidally evolve along. From Fig. 1, binary systems with smaller secondaries can clearly attain much wider separations than binary systems with components of similar size as predicted from the angular momentum limit in Eq. 5. Larger amounts of angular momentum are required to support systems with larger secondaries, and as angular momentum increases, the synchronous orbit curves surround one another with the inner synchronous orbit moving inward while the outer synchronous orbit moves outward approaching the angular momentum limit for smaller mass ratios.

Figure 1 is a hybrid of the "universal diagram" for tidal evolution of Counselman (1973) and Fig. 1 of Weidenschilling et al. (1989) in that it combines the ability to directly read off the fully synchronous orbits for specific values of angular momentum of Counselman with the mass ratio dependence of Weidenschilling et al. Furthermore, our figure accounts for the spin of the secondary, which Counselman does not, and can be modified to include tidal evolution timescales as done by Weidenschilling et al., which we will do in Section 5. Understanding evolution through our version of the universal diagram is aided by Fig. 2, which illustrates the general trends of tidal evolution when the spins of the components have the same sense of rotation as the motion in the mutual orbit, i.e., all motion is prograde. If the configuration of the system falls between the two solutions to the fully synchronous orbit equation for the $q$ of the system, meaning it visually appears "below" the fully synchronous orbit curve in $\left(q, a / R_{\mathrm{p}}\right)$-space like the lower arrow in Fig. 2, the system will evolve outward as spin angular momentum is transferred to the mutual orbit via the tidal interaction $\left(\omega_{\mathrm{p}}>n, \omega_{\mathrm{s}} \sim n\right)$. If the system sits "above" its fully synchronous orbit curve in the position of one of the upper arrows in Fig. 2, due to having a large secondary or a wide separation, the orbital angular momentum makes up a larger fraction of the total angular momentum such that the angular momentum available in the spins of the components requires that $\omega_{\mathrm{p}}<n$ (for $\omega_{\mathrm{s}} \sim n$ ). The system must then evolve inward as angular momentum from the orbit is transferred to the spins of the components. For systems with constant angular momentum, the cases of inward evolution require a binary formation mechanism that initially produces well-separated components with rotation rates slower than the mean motion because tidal evolution cannot have evolved a system outward to these configurations above the fully synchronous orbit curve. Note that binaries with equal-mass $(q=1)$ components in Fig. 1 only have fully synchronous end states if $J / J^{\prime}>0.44$. For $J / J^{\prime}<0.44$, cases can exist where $q$ lies entirely above the fully synchronous orbit curve (the uppermost arrow in Fig. 2) meaning the fully synchronous orbit equation has no solution for that value of $q$. The resulting inward tidal evolution may be important in the context of forming contact binary asteroids (Tavlor, 2009) and will be addressed in the future.

\section{Stability and $E=0$ limits of fully synchronous orbits}

The fully synchronous orbit solutions to Eq. 7 are equivalently thought of as contours of constant $J / J^{\prime}$ in $\left(q, a / R_{\mathrm{p}}\right)$-space satisfying:

$$
\frac{J}{J^{\prime}}=\frac{1}{\left(1+\frac{\rho_{\mathrm{p}}}{\rho_{\mathrm{s}}} q\right)^{1 / 6}} \frac{q}{(1+q)^{2}}\left(\frac{a}{R_{\mathrm{p}}}\right)^{1 / 2}+\alpha_{\mathrm{p}} \frac{1+\frac{\alpha_{\mathrm{s}}}{\alpha_{\mathrm{p}}}\left(\frac{\rho_{\mathrm{p}}}{\rho_{\mathrm{s}}}\right)^{2 / 3} q^{5 / 3}}{\left(1+\frac{\rho_{\mathrm{p}}}{\rho_{\mathrm{s}}} q\right)^{1 / 6}(1+q)}\left(\frac{a}{R_{\mathrm{p}}}\right)^{-3 / 2} .
$$

The maxima of the set of contour curves for a continuous range of $J / J^{\prime}$ are traced out by taking 


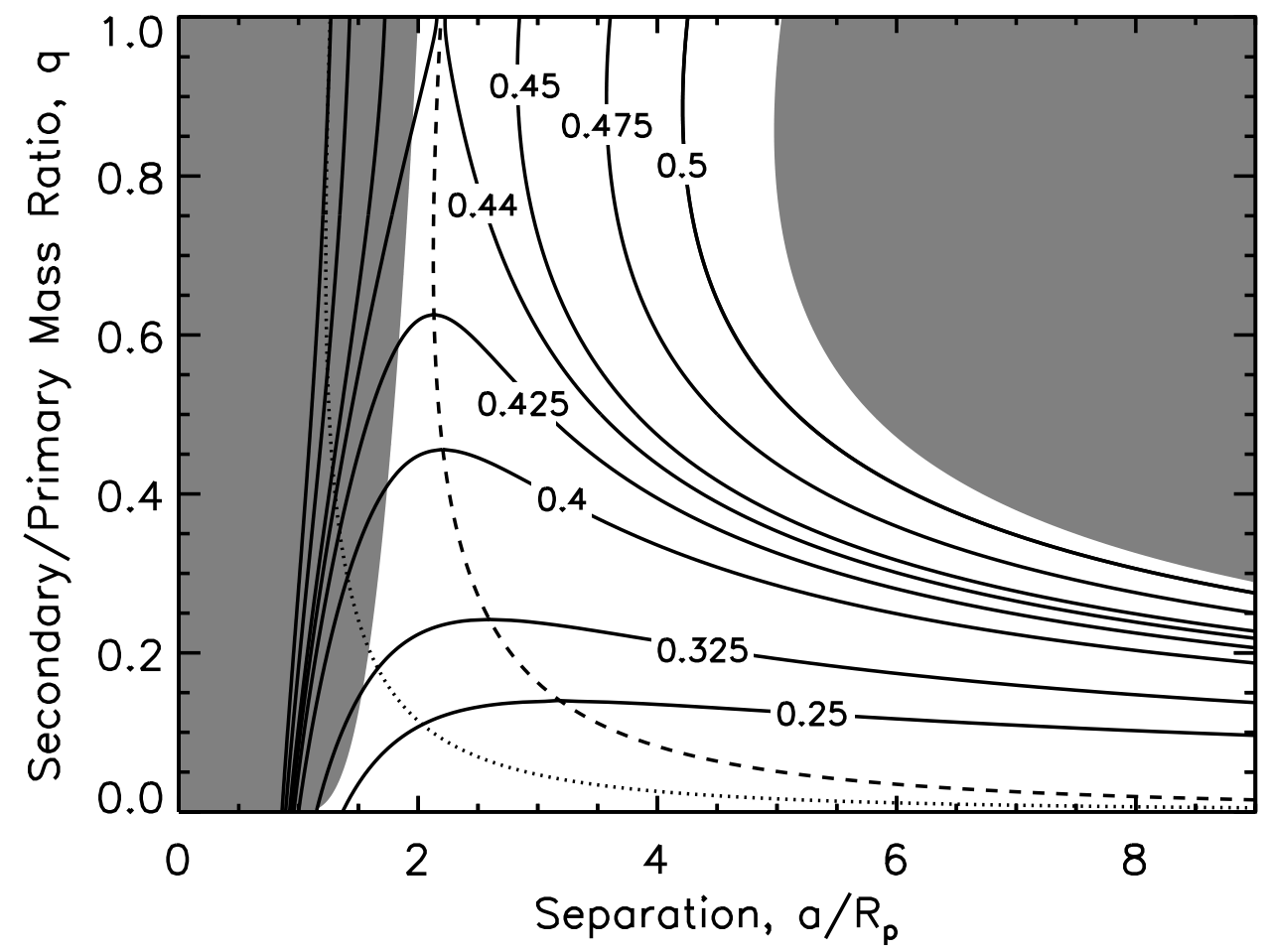

Fig. 1. Component separations $a_{\text {sync }} / R_{\mathrm{p}}$ for the fully synchronous orbits of binary systems of given a mass ratio $q$ and scaled angular momentum $J / J^{\prime}$. Solid lines indicate the solutions to Eq. 8 for $J / J^{\prime}=0.25,0.325,0.4,0.425,0.44,0.45,0.475$, and 0.5 . The shaded regions indicate separations that are inaccessible to the binary components. The region at left (Eq. 4) requires the components to be in contact, while the region at right (Eq. 5 , shown only for $J / J^{\prime}=0.5$ ) is disallowed by angular momentum conservation for circular orbits. The dashed line indicates the synchronous stability limit (see Section 4) that divides the two solutions for each mass ratio, if they exist, into an unstable inner synchronous orbit and a stable outer synchronous orbit. In most cases, the unstable inner synchronous orbit is within the contact limit. The dotted line indicates where fully synchronous systems have total energy $E=0$ (also see Section 4). 


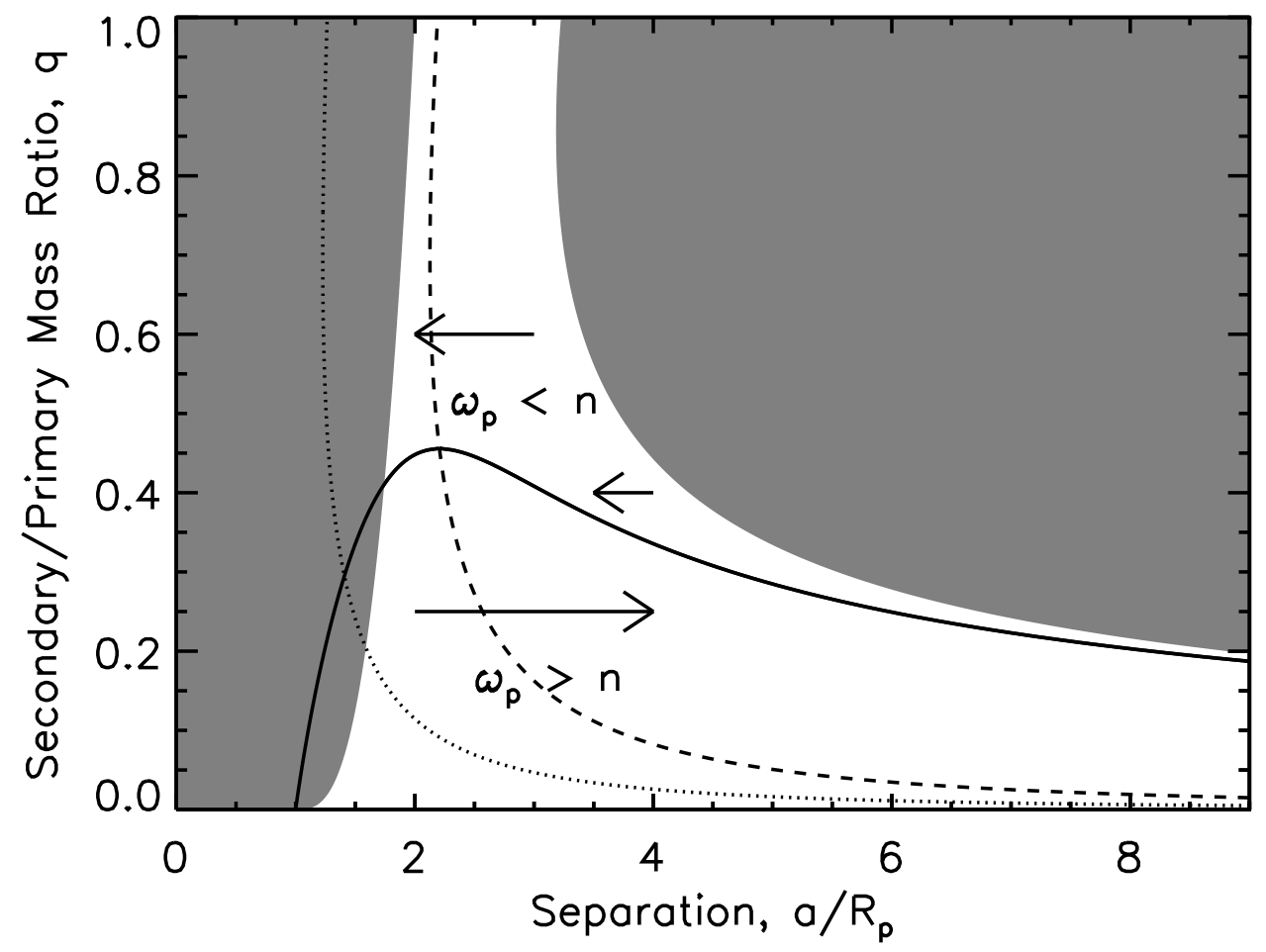

Fig. 2. Directions of tidal evolution for systems with $J / J^{\prime}=0.4$. For binaries dominated by the spin angular momentum of the primary mass, systems under the fully synchronous orbit curve will evolve outward as angular momentum is transferred from the rapid spin of the primary to the orbit $\left(\omega_{\mathrm{p}}>n\right)$. Systems above the fully synchronous orbit curve evolve inward as angular momentum is transferred from the orbit to the spins of the components $\left(\omega_{\mathrm{p}}<n\right)$ until either the outer synchronous orbit is reached or the orbit collapses to contact. The shaded regions indicate separations that are inaccessible to the binary components due to the contact limit and angular momentum limit for $J / J^{\prime}=0.4$. The dashed line indicates the synchronous stability limit, while the dotted line indicates where $E=0$ for fully synchronous orbits (see Section 4). 
the derivative of Eq. 9 with respect to $a / R_{\mathrm{p}}$ giving:

$$
\frac{a_{\text {stab }}}{R_{\mathrm{p}}}=\left[3 \alpha_{\mathrm{p}} \frac{1+q}{q}\left(1+\frac{\alpha_{\mathrm{s}}}{\alpha_{\mathrm{p}}}\left(\frac{\rho_{\mathrm{p}}}{\rho_{\mathrm{s}}}\right)^{2 / 3} q^{5 / 3}\right)\right]^{1 / 2} .
$$

For spherical components with similar uniform densities:

$$
\frac{a_{\mathrm{stab}}}{R_{\mathrm{p}}}=\left[\frac{6}{5} \frac{1+q}{q}\left(1+q^{5 / 3}\right)\right]^{1 / 2},
$$

which is the dashed curve in Fig. 1 that splits the synchronous orbit curves into inner and outer solutions when there are two real solutions to Eq. 8, Harris and Ward (1982) showed that Eq. 10 is also a stability limit against perturbations to the system such that the outer synchronous orbit in the two-sphere case that satisfies:

$$
\frac{a}{R_{\mathrm{p}}}>\left[\frac{6}{5} \frac{1+q}{q}\left(1+q^{5 / 3}\right)\right]^{1 / 2}
$$

is stable, while the inner synchronous orbit is not, meaning that the binary system will tend to evolve away from the inner synchronous orbit given the opportunity. If perturbed outward from the inner synchronous orbit, the components will tidally evolve outward to the stable outer synchronous orbit (the lower arrow in Fig. 2); if perturbed inward, the components will fall to contact, though for most $J / J^{\prime}$, the inner synchronous orbit is already within the contact limit. The arrows in Fig. 2 also show that perturbation of the system from the outer synchronous orbit will allow the system to return to the outer synchronous orbit, indicating its stability against tidal perturbation. It is also clear from Figs. 1] and 2, that the stability limit is always beyond the contact limit such that whenever a particle is lofted from the surface of the primary or when a parent body fissions, the components are initially in a tidally unstable configuration that may lead to re-impact (Scheeres, 2009).

For bodies with $\alpha_{\mathrm{p} . \mathrm{s}} \neq 2 / 5$, one may use the parameter $\lambda_{\mathrm{p}, \mathrm{s}}=5 \alpha_{\mathrm{p}, \mathrm{s}} / 2$ introduced by Descamps and Marchis (2008) to find that the stable outer synchronous orbit is described by:

$$
\frac{a}{R_{\mathrm{p}}}>\left[\frac{6}{5} \frac{1+q}{q}\left(\lambda_{\mathrm{p}}+\lambda_{\mathrm{s}} q^{5 / 3}\right)\right]^{1 / 2},
$$

correcting both the formula and direction of the inequality given by Marchis et al. (2008a,,$\underline{b})$. We note, though, that if one (or both) of the components of the binary system is nonspherical, Eq. 13 is not completely accurate because the formulation of the fully synchronous orbit curves in Eq. 7 . and thus the stability limit, would be complicated by the mutual gravitational potential between nonspherical bodies (Scheeres, 2009) and the synchronous rotation rate required for relative equilibrium (see Scheeres, 2006, 2007a). Therefore, Eq. 13 is exact only in the two-sphere case with $\alpha_{\mathrm{p}, \mathrm{s}} \neq 2 / 5$ due to density inhomogeneity within the individual components.

The stability limit in Eq. 10 gives the minimum separation of a stable, fully synchronous tidal end state for a given mass ratio $q$. The corresponding minimum angular momentum required for a system to have a stable tidal end state results from substituting Eq. 10 into Eq. 9 and, by assuming similar component densities and $\alpha_{\mathrm{p}, \mathrm{s}}=2 / 5$, can be written as: 


$$
J / J_{\text {crit }}^{\prime}=\left(\frac{512}{135}\right)^{1 / 4} \frac{q^{3 / 4}}{(1+q)^{23 / 12}}\left(1+q^{5 / 3}\right)^{1 / 4} .
$$

This critical value for the angular momentum is equivalent to that derived by Hut (1980) in the context of binary star systems. Satisfying the stability condition is a necessary, but not sufficient, condition for a stable end to tidal evolution. Systems that do not satisfy the stability condition have not reached a stable tidal end state, while systems that do satisfy the stability condition may or may not continue their evolution depending on the angular momentum of the system. At the minimum angular momentum of Eq. 14, the synchronous orbit equation (Eq. 8) has one degenerate solution, which is simply the stability limit, and tidal evolution may cease. If the angular momentum of the system exceeds $J / J_{\text {crit }}^{\prime}$, the synchronous orbit equation has two solutions, and the system will continue to evolve to the outer synchronous orbit. Of course, having less than $J / J_{\text {crit }}^{\prime}$ will cause the system to collapse as the synchronous orbit equation has no solution regardless of whether or not the stability condition is satisfied. This argument is used by Levrard et al. (2009) to posit that nearly all transiting extrasolar planets, in the absence of a parking mechanism, will inevitably collide with their host stars because these planetary systems lack sufficient angular momentum to support stable tidal end states for their components.

Upon full synchronization $\left(\omega_{\mathrm{p}}=\omega_{\mathrm{s}}=n\right)$, the total energy of the system (Eq. 6) may be positive or negative with the $E=0$ limit occurring at:

$$
\frac{a_{\mathrm{E}}}{R_{\mathrm{p}}}=\left[\alpha_{\mathrm{p}} \frac{1+q}{q}\left(1+\frac{\alpha_{\mathrm{s}}}{\alpha_{\mathrm{p}}}\left(\frac{\rho_{\mathrm{p}}}{\rho_{\mathrm{s}}}\right)^{2 / 3} q^{5 / 3}\right)\right]^{1 / 2},
$$

where spherical shapes have been assumed in determining the mutual gravitational potential. Fully synchronous orbits with semimajor axes $a_{\text {sync }}>a_{\mathrm{E}}$ have $E<0$ because the combination of translational and potential energy $\left(\propto a^{-1}\right)$ falls off more slowly with increasing $a$ than the rotational energy of the components $\left(\propto a^{-3}\right)$ when synchronized. Fully synchronous orbits with $a_{\text {sync }}<a_{\mathrm{E}}$ have $E>0$ and could evolve to escape given sufficient perturbation from relative equilibrium (Bellerose and Scheeres, 2008). Note that in the two-sphere case $a_{\mathrm{E}}=a_{\mathrm{stab}} / \sqrt{3}$ such that all stable, fully synchronous orbits have $E<0$, but the converse, having $E<0$, does not necessarily guarantee stability. For systems with $J / J^{\prime}>0.334$ in Fig. 1, the realistic fully synchronous orbit solutions, i.e., those beyond the contact limit, have $E<0$. Systems with less angular momentum can have unstable inner synchronous orbits for which $E>0$.

As an interesting side note, if one inserts $a_{\mathrm{E}}$ into $L$ and $S$ in Eq. 1 and Eq. 2 for a fully synchronous orbit, one finds that $L / S=1$ or the angular momentum of the system revolving about its center of mass is equal to the rotational angular momentum of the components (Scheeres, 2009). Similarly, inserting $a_{\text {stab }}$ finds $L / S=3$ or the angular momentum of the system's revolution is three times the rotational angular momentum (Hut, 1980; Scheeres, 2009). Thus, a stable fully synchronous tidal end state must satisfy $L / S \geq 3$ or, equivalently, the orbital angular momentum must account for more than $3 / 4$ of the total angular momentum of the system, which is observed in nearly equal-mass binary systems (90) Antiope (Michałowski et al., 2004; Descamps et al., 2007) and (69230) Hermes (Margot et al., 2006). 


\section{Material properties of binary asteroids}

During the tidal evolution process, angular momentum is transferred between the spins of the components $\omega_{\mathrm{p}}$ and $\omega_{\mathrm{s}}$ and the mutual orbit about the center of mass of the system. For a prograde system, angular momentum conservation (e.g., Murray and Dermott, 1999; Taylor and Margot, 2010) requires that the change with time of the orbital separation of the components follows:

$$
\begin{aligned}
\frac{\dot{a}}{R_{\mathrm{p}}}= & \frac{8 \sqrt{3}}{19} \frac{\pi^{3 / 2} G^{3 / 2} \rho_{\mathrm{p}}^{5 / 2} R_{\mathrm{p}}^{2}}{\mu_{\mathrm{p}} Q_{\mathrm{p}}} q(1+q)^{1 / 2}\left(\frac{a}{R_{\mathrm{p}}}\right)^{-11 / 2} \\
& \times\left[\operatorname{sign}\left(\omega_{\mathrm{p}}-n\right)+\frac{R_{\mathrm{s}}}{R_{\mathrm{p}}} \frac{\mu_{\mathrm{p}} Q_{\mathrm{p}}}{\mu_{\mathrm{s}} Q_{\mathrm{s}}} \operatorname{sign}\left(\omega_{\mathrm{s}}-n\right)\right] .
\end{aligned}
$$

The sign of the quantity $\omega-n$ for each component determines whether the separation increases or decreases due to tides raised on that component; if $\omega>n(\omega<n)$, tides slow (accelerate) the rotation of the component and increase (decrease) the orbital separation. The contribution from tides raised on the secondary are a factor of the size ratio weaker than from tides raised on the primary and, furthermore, the smaller secondary will tend to synchronize to the mean motion $n$ before the primary causing the tidal torque on the secondary, as well as its contribution to the change in orbital separation, to vanish earlier. The overall strength of the tidal mechanism is inversely related to the product $\mu Q$, which combines the rigidity or shear modulus $\mu$ of the materia 3 and the tidal dissipation function $Q$ that relates to the lag angle between the axis of symmetry of the tidal bulge and the tide-raising body (see Goldreich, 1963; Efroimsky and Williams, 2009, for detailed discussions). The larger the quantity $\mu Q$, the more resistant the material is to orbit evolution due to the tidal mechanism. Here, for completeness, we have allowed for the primary and secondary to have their own $\mu Q$ values.

\subsection{Caveats}

In estimating the material properties of binary asteroids, we assume that tides are the dominant method of dynamical evolution in these systems, specifically in the evolution of the separation between the components. It has been argued that the binary YORP effect (BYORP; Ćuk and Burns, 2005; Ćuk and Nesvorný, 2010; McMahon and Scheeres, 2010a), where a synchronous secondary acts to asymmetrically re-radiate sunlight with respect to its orbital velocity so that the orbit is expanded or contracted, can act on timescales faster than tidal evolution (Ćuk and Burns, 2005; Goldreich and Sari, 2009; McMahon and Scheeres, 2010b). This mechanism is similar to the YORP effect (Rubincam, 2000), an asymmetric re-radiation of sunlight with respect to rotational velocity. However, unlike the YORP effect (Tavlor et al., 2007; Lowry et al., 2007; Kaasalainen et al., 2007), BYORP has yet to be proven observationally. Furthermore, BYORP requires a synchronous secondary and thus cannot be the dominant active evolution mechanism for systems with asynchronous secondaries. Also of interest, specifically

\footnotetext{
${ }^{3}$ In deriving Eq. 16, it is assumed that for both components the rigidity $\mu$ dominates the stress due to self-gravity $\rho g R \sim G \rho^{2} R^{2}$, where $g$ is the surface gravity, which is reasonable for bodies under $200 \mathrm{~km}$ in radius (Weidenschilling et al., 1989) such that the potential Love number $k_{2} \propto \mu^{-1}$.
} 
among small binary asteroids with primaries less than $10 \mathrm{~km}$ in diameter, is the idea of mass lofting or "tidal saltation" (Harris et al., 2009), where particles at the equator of a rapidly spinning primary become weightless due to the gravitational presence of the secondary passing overhead, briefly enter orbit, and transfer angular momentum to the orbit of the secondary before falling back to the surface of the primary. This method has also been argued to expand the mutual orbit more quickly than tidal evolution (Fahnestock and Scheeres, 2009). It may be a combination of effects, also including close planetary flybys (Farinella, 1992; Bottke and Melosh, 1996a, b), that evolve the separation of near-Earth binary components, but tides are the only mechanism one can say at this time must act on all systems. For main-belt binaries with 100-km-scale primaries, tides should be the dominant mechanism because close planetary encounters are not feasible in

the main belt, the thermal YORP and BYORP effects are weakened by the increased heliocentric distance to the main belt (and the increased sizes of the bodies involved), and the primaries do not rotate rapidly enough nor are the secondaries close enough to produce mass lofting.

\subsection{Estimation of $\mu Q$}

Integration of Eq. 16 provides, symbolically, the evolution of the orbital separation as a function of time in terms of the product $\mu_{\mathrm{p}} Q_{\mathrm{p}}$ and the time over which tidal evolution has taken place $\Delta t$. Both $\mu_{\mathrm{p}} Q_{\mathrm{p}}$ and $\Delta t$ are inherent unknowns in any binary system, but their ratio is fully determined by the current separation of the binary system $a / R_{\mathrm{p}}$, the physical properties of the components, and the assumption of an initial separation of the components after the formation of the binary. The ratio is very weakly dependent on the initial separation (see Section 5.6); we choose $2 R_{\mathrm{p}}$ because it is both the contact limit for equal-size components and a reasonable initial separation for binaries formed via spin-up (Walsh et al., 2008). Thus, the evolution of the orbital separation gives:

$$
\frac{\mu_{\mathrm{p}} Q_{\mathrm{p}}}{\Delta t}=\frac{52 \sqrt{3}}{19} \pi^{3 / 2} G^{3 / 2} \rho_{\mathrm{p}}^{5 / 2} R_{\mathrm{p}}^{2} q(1+q)^{1 / 2}\left[\left(\frac{a}{R_{\mathrm{p}}}\right)^{13 / 2}-2^{13 / 2}\right]^{-1}\left(1+\frac{R_{\mathrm{s}}}{R_{\mathrm{p}}} \frac{\mu_{\mathrm{p}} Q_{\mathrm{p}}}{\mu_{\mathrm{s}} Q_{\mathrm{s}}} \frac{\tau_{\mathrm{s}}}{\Delta t}\right)
$$

for a system where tides on both components are causing the mutual orbit to expand with $\tau_{\mathrm{s}}$ representing the length of time the secondary contributes to the tidal evolution, which is less than or equal to the age of the binary system $\Delta t$. Ignoring the rightmost term in parentheses for the moment, to estimate either $\mu_{\mathrm{p}} Q_{\mathrm{p}}$ or $\Delta t$, the other must be assumed, where varying one by, say, an order of magnitude varies the other parameter by the same amount. With a judicious assumption of the age of the binary, one can estimate $\mu_{\mathrm{D}} Q_{\mathrm{p}}$ for the primary or at least place rough bounds on its value (Margot et al., 2002; Margot and Brown, 2003); similarly, by assuming a value of $\mu_{\mathrm{p}} Q_{\mathrm{p}}$, one can estimate the age of the binary (Walsh and Richardson, 2006; Marchis et al., 2008a.,b; Goldreich and Sari, 2009). A related analysis was done for the martian moon Phobos by Yoder (1982), who estimated its $\mu Q$ is at least of order $10^{11} \mathrm{~N} \mathrm{~m}^{-2}$ based on the eccentricity evolution of Phobos within the $\Delta t$ inferred from the minimum age of its surface.

If one ignores the contribution of the secondary, equivalent to setting $\tau_{\mathrm{s}}$ to zero, Eq. 17 represents a lower bound on $\mu_{\mathrm{p}} Q_{\mathrm{p}}$ for a given timescale $\Delta t$. Allowing the secondary to contribute 
for the entire age of the binary by letting $\tau_{\mathrm{s}} \rightarrow \Delta t$ gives an upper bound 4 on $\mu_{\mathrm{p}} Q_{\mathrm{p}}$, which is a factor of $1+\left(R_{\mathrm{s}} / R_{\mathrm{p}}\right)\left(\mu_{\mathrm{p}} Q_{\mathrm{p}} / \mu_{\mathrm{s}} Q_{\mathrm{s}}\right)$ greater than the lower bound found using only the primary. If $\Delta t$ is correct, the actual $\mu_{\mathrm{p}} Q_{\mathrm{p}}$ should lie between the two bounds as the secondary tends to be synchronously rotating such that $\tau_{\mathrm{s}}<\Delta t$ rather than contributing to the mutual orbit expansion over the entire age of the system. Ideally, one would know when the secondary became synchronized, evolve the system using both tides until $\tau_{\mathrm{s}}$, then include only the tide on the primary for the remainder of $\Delta t$. Our ignorance of if/when the secondary became synchronous cannot change the estimate of $\mu_{\mathrm{p}} Q_{\mathrm{p}}$ by more than a factor of $1+\left(R_{\mathrm{s}} / R_{\mathrm{p}}\right)\left(\mu_{\mathrm{p}} Q_{\mathrm{p}} / \mu_{\mathrm{s}} Q_{\mathrm{s}}\right)$, which is nominally less than two for components with equivalent material properties, far less critical than the choice of $\Delta t$, the age of the binary, but similar to the effect of measurement errors on the physical parameters in Eq. 17 (see Section 5.6).

In examining orbit expansion by tides, one cannot directly determine whether the primary and secondary have different $\mu Q$ values, though having a stronger or weaker secondary can change the estimate of $\mu_{\mathrm{p}} Q_{\mathrm{p}}$. Assuming equivalent $\mu Q$ values for the components, $\mu_{\mathrm{p}} Q_{\mathrm{p}}$ cannot be affected by more than a factor of two by accounting for the secondary; often the factor will be much less due to the size ratio of the components and the fact that synchronous secondaries require $\tau_{\mathrm{s}}<\Delta t$. If the secondary is mechanically stronger than the primary such that $\mu_{\mathrm{s}} Q_{\mathrm{s}}>\mu_{\mathrm{p}} Q_{\mathrm{p}}$, the effect on $\mu_{\mathrm{p}} Q_{\mathrm{p}}$ compared to ignoring the secondary in Eq. 17 must still be less than a factor of two because $\left(R_{\mathrm{s}} / R_{\mathrm{p}}\right)\left(\mu_{\mathrm{p}} Q_{\mathrm{p}} / \mu_{\mathrm{s}} Q_{\mathrm{s}}\right)\left(\tau_{\mathrm{s}} / \Delta t\right)<1$. Thus, having a stronger secondary than the primary serves to reduce the upper bound on $\mu_{\mathrm{p}} Q_{\mathrm{p}}$ for a specific age $\Delta t$, but the actual material strength of the secondary is not constrained. On the other hand, if the secondary is mechanically weaker than the primary such that $\mu_{\mathrm{s}} Q_{\mathrm{s}}<\mu_{\mathrm{p}} Q_{\mathrm{p}}$, depending on the size and $\mu Q$ ratios of the components and the age of the system $\Delta t$, the effect on $\mu_{\mathrm{p}} Q_{\mathrm{p}}$ compared to ignoring the secondary could conceivably exceed a factor of two, but is unlikely to differ by more than an order of magnitude unless the system is very young and the secondary is orders of magnitude weaker than the primary 5

For a synchronous secondary, the eccentricity can increase or decrease depending on how $\mu_{\mathrm{s}} Q_{\mathrm{s}}$ compares to $\mu_{\mathrm{p}} Q_{\mathrm{p}}$ (Goldreich, 1963; Goldreich and Soter, 1966) with the prediction that, for equivalent material properties, systems with monolithic components and $q \leq 0.31$ will have the eccentricity excited via tides (Harris and Ward, 1982; Weidenschilling et al., 1989). Given we observe the vast majority of binaries, including those with much smaller mass ratios than 0.31 , have circular mutual orbits, to prevent eccentricity excitation the secondaries must be mechanically weaker than the primaries (Margot and Brown, 2003) by roughly an order of magnitude to lower the limiting mass ratio from 0.31 to below the observed mass ratios in the binary population, e.g., by a factor of 30 for the smallest mass-ratio system we will discuss, (702) Alauda. However,

\footnotetext{
${ }^{4}$ We do not use the terms "lower bound" and "upper bound" in a rigorous mathematical sense here because factors such as uncertainties in the physical properties used in Eq. 17 can affect the values of these bounds by factors of order unity.

${ }^{5}$ The time it takes for the secondary to synchronize $\tau_{\mathrm{s}}$ shortens compared to $\Delta t$ as $\mu_{\mathrm{s}} Q_{\mathrm{s}}$ is made weaker so that $\tau_{\mathrm{s}} / \Delta t$ tends to cancel out the effect of $\mu_{\mathrm{p}} Q_{\mathrm{p}} / \mu_{\mathrm{s}} Q_{\mathrm{s}}$ growing. Only if $\tau_{\mathrm{s}}$ and $\Delta t$ are comparable due to the youth of the system will the disparity between the mechanical strength of the primary and secondary have a strong effect on the calculation at hand (if it can overcome the size ratio factor as well).
} 
Goldreich and Sari (2009) suggest that binary systems with gravitational-aggregate components, rather than monolithic ones, do not require much weaker secondaries to have circular mutual orbits as the eccentricity will damp for all mass ratios if the components are aggregates of similar material. So again, it is difficult to constrain the separate values of $\mu Q$ for the components, but our ignorance should not strongly affect our results for particular systems unless the extreme case of a very young binary with components having very disparate material properties exists. Therefore, when estimating the material properties of a binary system via Eq. 17, we may ignore the possibility of the components having different material properties to obtain an order-of-magnitude result 6 In the following sections we will also ignore the contribution of the secondary in Eq. 17 to find a lower bound for $\mu Q$ of the system with a specific age $\Delta t$. An upper bound on $\mu Q$ from accounting for the secondary is nominally less than a factor of two larger if the material properties of the components are similar.

\subsection{Large main-belt binaries}

First we consider binary systems in the main asteroid belt and among the Jupiter Trojans with 100-km-scale primary components that have been characterized by direct adaptive-optics imaging and lightcurve photometry. To be included in Table 1, orbital properties must be known and some estimate of the sizes must be available. Most of the binary systems discovered via imaging of large main-belt asteroids and Jupiter Trojans have secondaries roughly one-tenth the size of their primaries $(q \sim 0.001)$ or smaller. These secondaries are likely the result of a sub-catastrophic impact on the parent body (i.e., SMATS, smashed target satellites, as described by Durda et al. (2004)), which is supported by the angular momentum budget of the systems. With the exception of (90) Antiope and (617) Patroclus, which have nearly equal-size components, the $100-\mathrm{km}$ scale main-belt binaries have $J / J^{\prime}$ values of roughly 0.2 , well below the $J / J^{\prime} \sim 0.4$ regime characteristic of binaries formed via a spin-up mechanism. Among the $q<0.1$ binaries, the average primary component spins with a period of roughly $6 \mathrm{~h}$, twice the rotational breakup period, yet accounts for $97 \%$ of the angular momentum of the system due to the large mass disparity between the components. Nearly equal-mass binary (90) Antiope (Merline et al., 2000; Michałowski et al., 2004; Descamps et al., 2007) has $J / J^{\prime} \sim 0.5$ that is more similar to a binary formed through spin-up than through a sub-catastrophic collision. However, the sheer size of the components, each more than $80 \mathrm{~km}$ in diameter, and its location in the main belt make it difficult for the YORP effect or close planetary encounters to explain how the (90) Antiope system originally formed. Nearly equal-mass binary (617) Patroclus (Merline et al., 2001; Marchis et al., 2006; Mueller et al., 2010) has $J / J^{\prime} \sim 0.8$, far larger than the other large main-belt binaries we consider, but near the upper limit for giant impacts of $J / J^{\prime}<0.8$ (Canup, 2005). Such a high angular momentum content is more similar to many of the Kuiper-belt binaries that may have formed via $n$-body capture (see Noll et al., 2008, for a review) rather than spin-up or collisions.

The $q<0.1$ binaries in the main belt are in the midst of a lengthy tidal evolution. As shown in Fig. 3, of the $q<0.1$ binaries, only (22) Kalliope has reached the synchronous stability limit, but even (22) Kalliope is very far from reaching the outer synchronous orbit that nearly

\footnotetext{
${ }^{6}$ By ignoring any possible difference in $\mu Q$ of the components, Eq. 17 gives the value of $\mu Q$ for the system, as in it applies to both components rather than the primary only.
} 


\begin{tabular}{|c|c|c|c|c|c|c|c|c|}
\hline & Name & $R_{\mathrm{p}}(\mathrm{km})$ & $R_{\mathrm{s}} / R_{\mathrm{p}}$ & $q$ & $\rho\left(\mathrm{g} \mathrm{cm}^{-3}\right)$ & $a / R_{\mathrm{p}}$ & $J / J^{\prime}$ & $\mu Q\left(\mathrm{~N} \mathrm{~m}^{-2}\right)$ \\
\hline 22 & Kalliope & 85 & 0.213 & 0.009664 & 2.5 & 12.5 & 0.22 & $3.3 \times 10^{12}$ \\
\hline 45 & Eugenia & 98 & 0.036 & 0.000047 & 1.1 & 12.1 & 0.19 & $3.4 \times 10^{9}$ \\
\hline 87 & Sylvia & 128 & 0.063 & 0.000250 & 1.5 & 10.6 & 0.20 & $1.6 \times 10^{11}$ \\
\hline 90 & Antiope $^{a}$ & 43 & 0.955 & 0.871 & 1.3 & 3.9 & 0.49 & $<4.0 \times 10^{16}$ \\
\hline 107 & Camilla & 103 & 0.050 & 0.000125 & 1.9 & 12.0 & 0.18 & $4.1 \times 10^{10}$ \\
\hline 121 & Hermione & 103 & 0.066 & 0.000287 & 1.1 & 7.5 & 0.22 & $5.1 \times 10^{11}$ \\
\hline 130 & Elektra & 90 & 0.026 & 0.000018 & 3.0 & 14.0 & 0.14 & $5.1 \times 10^{9}$ \\
\hline 283 & Emma & 73 & 0.079 & 0.000493 & 0.8 & 8.2 & 0.20 & $1.1 \times 10^{11}$ \\
\hline 617 & Patroclus $^{b}$ & 51 & 0.920 & 0.779 & 1.3 & 13.5 & 0.83 & $1.5 \times 10^{13}$ \\
\hline 702 & Alauda & 97 & 0.018 & 0.000006 & 1.6 & 12.6 & 0.13 & $7.9 \times 10^{8}$ \\
\hline 762 & Pulcova & 67 & 0.160 & 0.004096 & 1.9 & 12.2 & 0.17 & $5.1 \times 10^{11}$ \\
\hline
\end{tabular}

Table 1: Physical properties of main-belt and Jupiter-Trojan binary asteroids with primary radii of order $100 \mathrm{~km}$. $R_{\mathrm{p}}$ is the radius of the primary; $R_{\mathrm{S}} / R_{\mathrm{p}}$ is the size ratio of the components; $q$ is the mass ratio, taken to be the size ratio cubed by assuming components of equal density $\rho ; a / R_{\mathrm{p}}$ is the semimajor axis of the mutual orbit; $J / J^{\prime}$ is the normalized angular momentum assuming spherical bodies and a synchronous secondary using Eqs. 1 and 2, for which an average value of roughly 0.2 for $q<0.1$ binaries indicates a sub-catastrophic collisional origin rather than formation through spin-up. For the triple systems (45) Eugenia and (87) Sylvia, only properties of the larger outer satellite are considered. Values of $\mu Q$ correspond to tides raised on the primary acting over the 4.5 Gyr age of the Solar System. Accounting for tides raised on the secondary would increase $\mu Q$ by no more than a factor of two. Data are taken from the Ondrejov Asteroid Photometry Project binary asteroid parameters table (http://www.asu.cas.cz/ asteroid/binastdata.htm, 2010 April 8 release) with the exception of (702) Alauda whose parameters are provided by Rojo and Margot (2011).

${ }^{a}$ (90) Antiope is known to be in a fully synchronous state where tidal evolution has ceased. Because of its nearly equal-size components in such close proximity, its tidal evolution occurred over much less than the age of the Solar System.

${ }^{b}$ (617) Patroclus is believed to be in a fully synchronous state and must have formed at a larger initial separation than the value of $2 R_{\mathrm{p}}$ assumed here. 
coincides with the angular momentum limit for these systems. The inner synchronous orbit for $J / J^{\prime}=0.2$ lies above the contact limit, so these binaries must have begun their tidal evolution from an initial separation of roughly $2 R_{\mathrm{p}}$ or more to have evolved outward due to tides. Nearly equal-mass binaries (90) Antiope (Michałowski et al., 2004; Descamps et al., 2007) and (617) Patroclus (Marchis et al., 2006; Mueller et al., 2010) are both believed to be in their fully synchronous tidal end states where the rotational periods of the components equal the period of the mutual orbit. Of the large main-belt binaries listed in Table 1, only (130) Elektra and (283) Emma have mutual orbits that are not roughly circular, each having an eccentricity $e \sim 0.1$ likely caused by tidal excitation (Marchis et al., 2008a).

Assuming the value of $\mu Q$ of $10^{11} \mathrm{~N} \mathrm{~m}^{-2}$ for Phobos determined by Yoder (1982) is applicable to the components of main-belt binaries, timescales for tidal evolution are plotted in Fig. 3 and illustrate the interplay between material strength and system age. Systems that plot to the right of the 4.5 Gyr curve, the age of the Solar System, must either have smaller $\mu Q$ values than Phobos or have evolved very little in separation since formation. Systems that plot to the left of the 4.5 Gyr curve are either younger than 4.5 Gyr or must have $\mu Q$ values larger than $10^{11} \mathrm{~N} \mathrm{~m}^{-2}$ if they are actually 4.5 Gyr old. Solid rock has a rigidity $\mu$ of $10^{10} \mathrm{~N} \mathrm{~m}^{-2}$ or greater (Goldreich and Soter, 1966; Dziewonski and Anderson, 1981) and, assuming $Q \gg 1$, the product $\mu Q$ for solid rock would be of order $10^{12} \mathrm{~N} \mathrm{~m}^{-2}$ or greater; Burns and Safronov (1973) adopt a $\mu Q$ value of $3 \times 10^{13}$ $\mathrm{N} \mathrm{m}^{-2}$ as an extreme case for solid, non-porous rock in their analysis of damping to principal axis rotation. Furthermore, Harris (1994) argues that $\mu Q$ should fall within a factor of 100 of $5 \times 10^{11} \mathrm{~N} \mathrm{~m}^{-2}$ based on analyses of Phobos and Comet Halley (Peale and Lissauer, 1989). In summary, a monolithic rock may have $\mu Q$ of order $10^{12} \mathrm{~N} \mathrm{~m}^{-2}$ or greater while a fractured rock would be expected to have a lower $\mu Q$ similar to that of Phobos with $\mu Q$ continuing to decrease with increased fracturing (He and Ahrens, 1994).

If instead of assuming a value of $\mu Q$ for the binary systems, we set $\Delta t$ to $4.5 \mathrm{Gyr}$, the age of the Solar System and the maximum amount of time these systems could have been tidally evolving, we can place an upper bound 8 on $\mu Q$ with Eq. 17. Ignoring (90) Antiope and (617) Patroclus, which can tidally evolve on timescales much more rapid than the age of the Solar System due to having nearly equal-size components, the median $\mu Q$ from Table 1 for main-belt binaries with $100-\mathrm{km}$-scale primaries is $1 \times 10^{11} \mathrm{~N} \mathrm{~m}^{-2}$, the same value estimated by Yoder (1982) for Phobos and reasonable for fractured rock. Individual values of $\mu Q$ are roughly consistent with the prediction of Harris (1994) and range over more than three orders of magnitude from $7.9 \times 10^{8} \mathrm{~N} \mathrm{~m}^{-2}$ for $(702)$ Alauda to $3.3 \times 10^{12} \mathrm{~N} \mathrm{~m}^{-2}$ for (22) Kalliope indicating a range of internal structures from heavily fractured or shattered rock to solid rock if all large main-belt binary systems are billions of years old. If a binary system is actually younger than $4.5 \mathrm{Gyr}, \mu Q$ will scale downward by the same factor.

Given the low $\mu Q$ values of (702) Alauda, (45) Eugenia, and (130) Elektra and the implied porosities of the primary components based on their densities in Table 1, some of the large

\footnotetext{
${ }^{7}$ When considering a specific value of $\Delta t$, Eq. 17 provides a lower bound when ignoring the secondary and an upper bound when accounting for the secondary over the entire age $\Delta t$. Here, we are considering all possible values of $\Delta t$ such that using the maximum possible age will give the maximum $\mu Q$ value to within a factor of two using Eq. 17 and accounting only for tides on the primary component.
} 


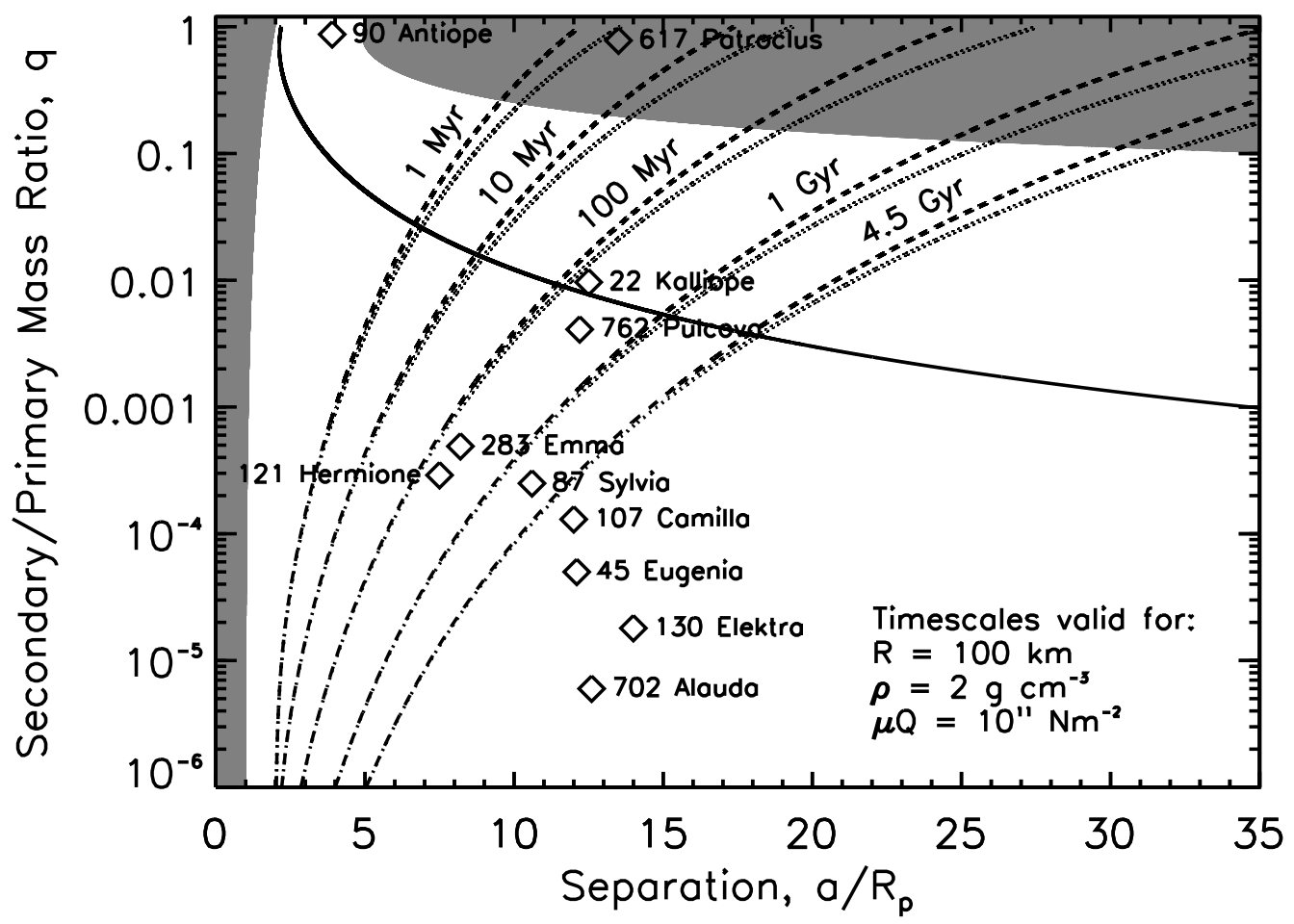

Fig. 3. Mass ratio $q$ and primary-secondary separation $a / R_{\mathrm{p}}$ for $100-\mathrm{km}$-scale main-belt and JupiterTrojan binaries along with the angular momentum limit for $J / J^{\prime}=0.5$. The solid curve is the stability limit. Tidal evolution timescales are plotted assuming $\rho_{\mathrm{p}, \mathrm{s}}=2 \mathrm{~g} \mathrm{~cm}^{-3}, R_{\mathrm{p}}=100 \mathrm{~km}$, and $\mu_{\mathrm{p}} Q_{\mathrm{p}}=\mu_{\mathrm{s}} Q_{\mathrm{s}}=10^{11} \mathrm{~N} \mathrm{~m}^{-2}$ and allowing tides raised on the primary only (dashed) or both components (dotted) to contribute over the entire evolution from an initial separation of $2 R_{\mathrm{p}}$. With these parameters, (90) Antiope evolves far more rapidly than the other main-belt binaries. 
main-belt primaries may be shattered and porous gravitational aggregates. In the gravitationalaggregate model of Goldreich and Sari (2009), void space (porosity) between the constituent particles, each of which has the rigidity of solid rock of order $10^{10} \mathrm{~N} \mathrm{~m}^{-2}$, and the increased stress at contact points lower the effective rigidity of the aggregate as a whole compared to monolithic rock 8 and can account for values of $\mu Q$ of order $10^{10}$ or $10^{11} \mathrm{~N} \mathrm{~m}^{-2}$ for gravitational aggregates $100 \mathrm{~km}$ in radius and assuming $Q \sim 10-100$. Since $\mu Q$ values calculated for (702) Alauda, (45) Eugenia, and (130) Elektra fall below $10^{10} \mathrm{~N} \mathrm{~m}^{-2}$, this could indicate that another mechanism is assisting tides in the semimajor-axis expansion of these systems, our ignorance of which results in a lower $\mu Q$ to account for the work done by the non-tidal process. If, instead, these systems formed near their current configurations, $\mu Q$ would correspondingly increase such that the system evolves in semimajor axis more slowly over the same time period. However, the dependence on initial separation is so weak (see Section [5.6) that the systems would have to have formed beyond $10 R_{\mathrm{p}}$ to raise $\mu Q$ above $10^{10} \mathrm{~N} \mathrm{~m}^{-2}$, which is difficult by sub-catastrophic collision. Durda et al. (2004) find that the material excavated during a sub-catastrophic collision that initially orbits close to the primary typically has a semimajor axis of $4-7 R_{\mathrm{p}}$, and further collisions among the orbiting material will eventually coalesce to form a single secondary orbiting within "several" primary radii rather than beyond $10 R_{\mathrm{p}}$.

The despinning timescales for the binary components of the $R_{\mathrm{s}} / R_{\mathrm{p}}<0.1(q<0.001)$ systems, assuming $\mu Q$ of $1 \times 10^{11} \mathrm{~N} \mathrm{~m}^{-2}$, are of order $1 \mathrm{Myr}$ for the secondaries (if they began their tidal evolution at an initial separation within a handful of primary radii) and much longer than the age of the Solar System for the primaries. Given the relatively short timescales for the secondaries, one would expect the secondaries in collisional binaries to have synchronized their rotation rate to their mean motion in the mutual orbit even if the systems are much younger than $4.5 \mathrm{Gyr}$ old. Because the despinning timescales of the primaries far exceed the age of the Solar System, however, the fact the systems are still tidally evolving does not place a limit on the age of the binaries. The rapid synchronization of the secondary and the negligible despinning of the primary make our ignorance of the initial spin states of the components unimportant for tidal evolution of binaries with small secondaries. In addition, the small size of the secondary with respect to the primary and its rapid despinning timescale compared to the assumed age of the binary also make the correction in Eq. 17 due to accounting for the secondary negligible $\left[\left(R_{\mathrm{s}} / R_{\mathrm{p}}\right)\left(\tau_{\mathrm{s}} / \Delta t\right) \sim 10^{-4}\right.$ compared a maximum possible contribution of unity]. We also note that the smallest secondaries in this subset are several kilometers in diameter, which gives them collisional lifetimes of more than 1 Gyr (Bottke et al., 2005) such that the existence of a secondary does not limit the age of a large main-belt binary either.

Taking the median $\mu Q$ of $1 \times 10^{11} \mathrm{~N} \mathrm{~m}^{-2}$ found for the $q<0.1$ large main-belt binaries, equal-mass binary (90) Antiope needs less than 10,000 years to evolve from near-contact to its present configuration. If the components of Antiope are heavily fractured gravitational aggregates, the time needed to tidally evolve becomes even shorter by an order of magnitude for every order of magnitude less than $10^{11} \mathrm{~N} \mathrm{~m}^{-2}$ the material strength of Antiope is. Even for monolithic rock

8 Goldreich and Sari (2009) find that the minimum rigidity of a gravitational aggregate compares to monolithic rock as $\mu \sim\left(8 \pi G \rho^{2} R^{2} \mu_{\text {monolith }} / 57 \epsilon_{\mathrm{Y}}\right)^{1 / 2} \sim 10^{7}(R / \mathrm{km}) \mathrm{N} \mathrm{m}^{-2}$, where $\epsilon_{\mathrm{Y}} \sim 10^{-2}$ is the yield strain. A gravitational aggregate $100 \mathrm{~km}$ in radius would have a minimum rigidity of order $10^{9} \mathrm{~N} \mathrm{~m}^{-2}$. 
with $\mu Q$ of order $10^{13} \mathrm{~N} \mathrm{~m}^{-2}$, Antiope can fully evolve by tides within 1 Myr. Though Antiope is a perfect example of the result of tidal evolution, as it resides in a stable fully synchronous tidal end state, the system can evolve to its observed state so rapidly for any reasonable $\mu Q$ value that one cannot constrain the material properties of Antiope well through tidal evolution. Timescales for (617) Patroclus are longer due to the wide separation of its components with tidal despinning acting on million-year timescales assuming the median $\mu Q$ value. Based on its total angular momentum, the Patroclus system must have formed with the components separated by at least $7 R_{\mathrm{p}}$ even if the components once rotated at the cohesionless breakup rate. However, this wider initial separation only affects the calculation of $\mu Q$ by $1 \%$ compared to our standard assumption of $2 R_{\mathrm{p}}$.

\subsection{Near-Earth binaries}

We also consider binary systems in the near-Earth region well-characterized by radar and lightcurve observations, all of which have primaries with diameters on the 1-km-scale or smaller. These systems are most likely the result of a spin-up mechanism (Margot et al., 2002; Richardson and Walsh, 2006; Descamps and Marchis, 2008) as evidenced by the $J / J^{\prime} \sim 0.4$ values for the near-Earth binaries in Table 2, Since the observational confirmation of the asteroidal YORP effect (Taylor et al., 2007; Lowry et al., 2007; Kaasalainen et al., 2007), spin-up via anisotropic thermal re-radiation of absorbed sunlight (Rubincam, 2000; Bottke et al., 2006) has become the preferred binary formation mechanism among $\mathrm{km}$-scale parent bodies in the near-Earth region and perhaps the main belt (Pravec and Harris, 2007). Further evidence of spin-up comes from the rapid rotation of the primaries that, ignoring nearly equal-mass binary (69230) Hermes, spin on average at $\sim 90 \%$ of their cohesionless breakup rate with a mean period of $2.8 \mathrm{~h}$ and contain over $80 \%$ of the angular momentum of the system in their spin. Though they spin faster than their large main-belt counterparts, the primaries of near-Earth binaries contain a smaller fraction of the angular momentum of the system owing to their larger secondaries. The YORP spin-doubling timescale, the time required for an asteroid to change its rotation period (or rotation rate) by a factor of two due to radiative torques, is of order $1 \mathrm{Myr}$ for a km-scale asteroid in the near-Earth region (Rubincam, 2000; Vokrouhlický and Čapek, 2002; Čapek and Vokrouhlický, 2004) allowing the parent body of a near-Earth binary system to spin-up rapidly and also allowing the post-formation primary component to maintain its rotation near the breakup rate.

Aside from nearly equal-mass binary (69230) Hermes that has reached its fully synchronous end state (Margot et al., 2006), the binaries in the near-Earth region are also in the midst of a lengthy tidal evolution. As shown by Fig. 4, a handful of systems have evolved past the stability limit, but all remain far from reaching the outer synchronous orbit for $J / J^{\prime}=0.4$ that lies at $8 R_{\mathrm{p}}$ for $q=0.2$ and rapidly recedes further out as $q$ decreases. Among those beyond the stability limit, (35107) $1991 \mathrm{VH}$ and (185851) $2000 \mathrm{DP}_{107}$ have been well studied. (35107) $1991 \mathrm{VH}$ is believed to be an asynchronous binary as three periods are sometimes detected in lightcurves (Pravec et al., 2006), presumably the mutual orbit period, the rotation of the primary, and the nonsynchronous rotation of the secondary. Tumbling of the primary (Pravec et al., 2006) or the secondary (Margot et al., 2008) could also explain the third periodicity. The orbit and rotation periods of the secondary of (185851) $2000 \mathrm{DP}_{107}$ suggest synchronization, but the primary has not been despun (Margot et al., 2002; Pravec et al., 2006). These systems illustrate the 


$\begin{array}{rlccccccc} & \text { Name } & R_{\mathrm{p}}(\mathrm{km}) & R_{\mathrm{s}} / R_{\mathrm{p}} & q & \rho\left(\mathrm{g} \mathrm{cm}^{-3}\right) & a / R_{\mathrm{p}} & J / J^{\prime} & \mu Q\left(\mathrm{~N} \mathrm{~m}^{-2}\right) \\ 3671 & \text { Dionysus } & 0.75 & 0.2 & 0.0080 & 2.0 & 5.3 & 0.35 & 7.2 \times 10^{7} \\ 5381 & \text { Sekhmet } & 0.5 & 0.3 & 0.0270 & 2.0 & 3.1 & 0.37 & 3.8 \times 10^{9} \\ 35107 & 1991 \mathrm{VH} & 0.6 & 0.38 & 0.0549 & 2.0 & 6.0 & 0.44 & 1.4 \times 10^{8} \\ 65803 & \text { Didymos } & 0.38 & 0.22 & 0.0106 & 2.0 & 3.0 & 0.41 & 9.8 \times 10^{8} \\ 66063 & 1998 \mathrm{RO}_{1} & 0.4 & 0.48 & 0.1106 & 2.0 & 3.6 & 0.47 & 3.8 \times 10^{9} \\ 66391 & 1999 \mathrm{KW}_{4} & 0.64 & 0.33 & 0.0359 & 2.0 & 4.0 & 0.37 & 1.6 \times 10^{9} \\ 69230 & \text { Hermes } & 0.3 & 0.9 & 0.7290 & 2.0 & 4.0 & 0.49 & 8.4 \times 10^{9} \\ 85938 & 1999 \mathrm{DJ}_{4} & 0.18 & 0.5 & 0.1250 & 2.0 & 4.1 & 0.49 & 3.7 \times 10^{8} \\ 88710 & 2001 \mathrm{SL}_{9} & 0.4 & 0.28 & 0.0220 & 2.0 & 3.8 & 0.40 & 5.2 \times 10^{8} \\ 137170 & 1999 \mathrm{HF}_{1} & 1.75 & 0.23 & 0.0122 & 2.0 & 3.4 & 0.40 & 1.1 \times 10^{10} \\ 164121 & 2003 \mathrm{YT}_{1} & 0.5 & 0.18 & 0.0058 & 2.0 & 6.4 & 0.40 & 6.6 \times 10^{6} \\ 175706 & 1996 \mathrm{FG}_{3} & 0.75 & 0.31 & 0.0298 & 2.0 & 3.7 & 0.29 & 2.7 \times 10^{9} \\ 185851 & 2000 \mathrm{DP}_{107} & 0.4 & 0.41 & 0.0689 & 1.7 & 6.6 & 0.48 & 2.9 \times 10^{7} \\ & 1994 \mathrm{AW}_{1} & 0.5 & 0.49 & 0.1176 & 2.0 & 4.8 & 0.50 & 9.7 \times 10^{8} \\ & 2000 \mathrm{UG}_{11} & 0.13 & 0.58 & 0.1951 & 2.0 & 4.3 & 0.43 & 2.2 \times 10^{8} \\ & 2002 \mathrm{CE}_{26} & 1.73 & 0.09 & 0.0007 & 0.9 & 2.7 & 0.43 & 4.4 \times 10^{8} \\ & 2004 \mathrm{DC}^{2} & 0.17 & 0.21 & 0.0093 & 1.7 & 4.4 & 0.41 & 9.6 \times 10^{6} \\ & 2005 \mathrm{AB} & 0.95 & 0.24 & 0.0138 & 2.0 & 4.0 & 0.29 & 1.3 \times 10^{9} \\ & 2005 \mathrm{NB}_{7} & 0.25 & 0.4 & 0.0640 & 2.0 & 3.7 & 0.34 & 7.5 \times 10^{8} \\ & 2006 \mathrm{GY}_{2} & 0.2 & 0.2 & 0.0080 & 2.0 & 3.0 & 0.37 & 2.2 \times 10^{8} \\ & 2007 \mathrm{DT}_{103} & 0.15 & 0.33 & 0.0359 & 2.0 & 3.6 & 0.39 & 1.6 \times 10^{8}\end{array}$

Table 2: Physical properties of near-Earth binary asteroids with primary radii of order $1 \mathrm{~km}$ or smaller. Densities are estimated as $2 \mathrm{~g} \mathrm{~cm}^{-3}$ (see Pravec and Harris, 2007, for details) unless the separation is known from radar observations, which allows the density to be calculated via Kepler's Third Law. $J / J^{\prime}$ in each case roughly satisfies the condition for binary formation via spin-up of a single parent body. Values of $\mu Q$ correspond to tides raised on the primary only acting over the 10 Myr dynamical lifetime of near-Earth asteroids. Accounting for tides raised on the secondary would increase $\mu Q$ by no more than a factor of two. Data are taken from the Ondrejov Asteroid Photometry Project binary asteroid parameters table (http://www.asu.cas.cz/ asteroid/binastdata.htm, 2010 April 8 release) with the exceptions of $2000 \mathrm{DP}_{107}$ from Margot et al. (2002) and $2004 \mathrm{DC}$ whose parameters are provided by the authors.

${ }^{a}$ (69230) Hermes is known to be in a fully synchronous state where tidal evolution has ceased. 
necessary, but not sufficient nature of crossing the stability limit for completing tidal evolution. Because the inner synchronous orbit is buried within the contact limit for the $q \sim 0.1$ binaries with $J / J^{\prime}=0.4$ (see Fig. 1), the secondaries have tidally evolved outward since the binaries were formed.

The dynamical lifetime of an asteroid in the near-Earth region is of order 10 Myr (Gladman et al., 1997, 2000), so the fact that all near-Earth binary systems lie beyond the generic 10 Myr evolution curve in Fig. 4 implies either the actual $\mu Q$ values are much weaker than the assumed $10^{11} \mathrm{~N} \mathrm{~m}^{-2}$ or that the systems must be older than 10 Myr having formed in the main belt prior to injection into the near-Earth region. Assuming all the binaries have tidally evolved for $10 \mathrm{Myr}$, the median $\mu Q$ in Table 2 is $5 \times 10^{8} \mathrm{~N} \mathrm{~m}^{-2}$, more than two orders of magnitude smaller than the median $\mu Q$ for the large main-belt binaries. A binary formed in the near-Earth region more recently than $10 \mathrm{Myr}$ ago requires a smaller $\mu Q$ than presented to complete the same tidal evolution. If formed in the main belt, the age of the binaries could extend to, at most, the collisional lifetime of a body $1 \mathrm{~km}$ in diameter, which is a few hundred million years (Bottke et al., 2005). Thus, the $\mu Q$ values in Table 2 could increase by roughly an order of magnitude if near-Earth binaries formed in the main belt.

For the median $\mu Q$ value of $5 \times 10^{8} \mathrm{~N} \mathrm{~m}^{-2}$, the secondaries in near-Earth binary systems are despun on 0.1-1 Myr timescales and thus are expected to be synchronously rotating for systems that are $10 \mathrm{Myr}$ old, while the primaries are despun on timescales closer to the dynamical or collisional lifetimes of the bodies. Nearly equal-mass binary (69230) Hermes needs less than 1 Myr to tidally evolve from near-contact to its fully synchronous end state assuming the median $\mu Q$ value. Because the YORP timescale for $\mathrm{km}$-scale asteroids in the near-Earth region is similar in magnitude to the tidal despinning timescale for the secondaries, YORP spin-up could hinder, negate, or even overcome tidal spin-down, delaying or preventing synchronous lock of the secondaries (Goldreich and Sari, 2009). The YORP timescale is much faster than the tidal despinning timescale for the primaries, which can help the primaries retain a rapid rotation (and possibly trigger a mass-lofting scenario) while tides evolve the separation of the components.

Though far less rigid than solid rock, $\mu$ as low as $10^{7} \mathrm{~N} \mathrm{~m}^{-2}$ for a body $1 \mathrm{~km}$ in radius $\left(10^{6}\right.$ $\mathrm{N} \mathrm{m}^{-2}$ for a body $100 \mathrm{~m}$ in radius) remain reasonable within the theory of Goldreich and Sari (2009) for gravitational-aggregate structure (see Footnote 8). Assuming $Q \sim 10-100$ is applicable, then gravitational aggregates can account for $\mu Q$ values of order $10^{8}$ or $10^{9} \mathrm{~N} \mathrm{~m}^{-2}$ for km-scale near-Earth binaries. Even at such low rigidities, $\mu$ still dominates over the stress due to self-gravity $g \rho R$ because of the low surface gravity $g$ and small size of the typical near-Earth asteroid.

For binary systems $2004 \mathrm{DC}$ and (164121) $2003 \mathrm{YT}_{1}$ with $\mu Q$ values below $10^{7} \mathrm{~N} \mathrm{~m}^{-2}$, the weakness of the bodies is difficult to reconcile with Goldreich and Sari's theory of gravitationalaggregate structure. To justify a more rigid structure with $\mu Q$ larger than we calculate in this tidal evolution scenario, one of the following must be true: the system formed recently in nearly its current configuration leaving $\mu Q$ unconstrained, the system is older than $10 \mathrm{Myr}$, or the orbit expansion is aided by another mechanism or mechanisms, e.g., BYORP, mass lofting, or close planetary flyby. Intriguingly, 2004 DC (Tavlor et al., 2008) and $2003 \mathrm{YT}_{1}$ (Nolan et al., 2004) are also both believed to have asynchronous secondaries that are not despun to the mean motion in their mutual orbit and have moderate eccentricities greater than 0.1 . These properties place them in the minority among near-Earth binary systems that tend to have synchronous secondaries and circularized mutual orbits. Given that the secondaries are rapidly despun by tides compared 


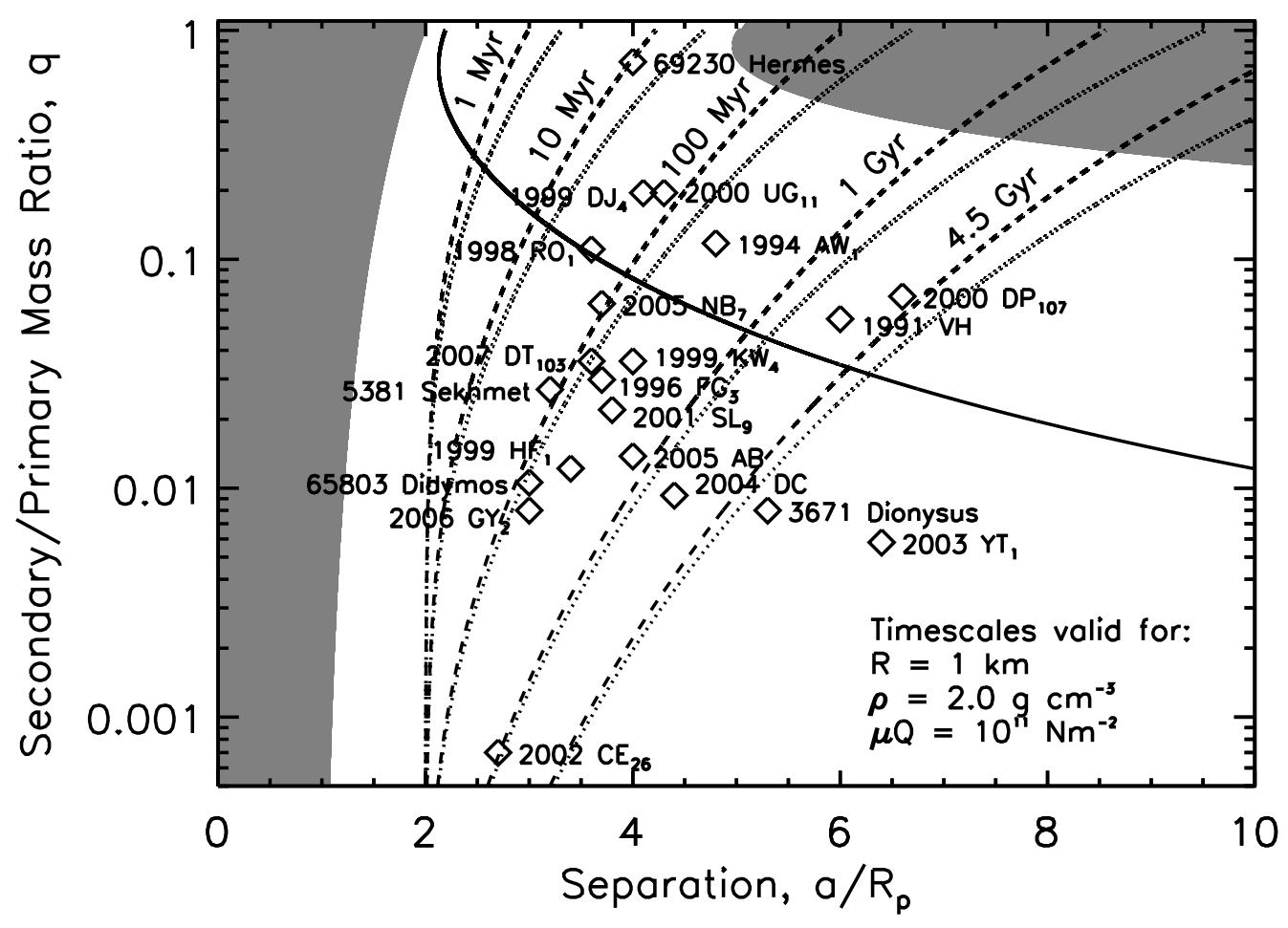

Fig. 4. Mass ratio $q$ and primary-secondary separation $a / R_{\mathrm{p}}$ for near-Earth binaries along with the angular momentum limit for $J / J^{\prime}=0.5$. The solid curve is the stability limit. Tidal evolution timescales are plotted assuming $\rho_{\mathrm{p}, \mathrm{s}}=2 \mathrm{~g} \mathrm{~cm}^{-3}, R_{\mathrm{p}}=1 \mathrm{~km}$, and $\mu_{\mathrm{p}} Q_{\mathrm{p}}=\mu_{\mathrm{s}} Q_{\mathrm{s}}=10^{11} \mathrm{~N} \mathrm{~m}^{-2}$ and allowing tides raised on the primary only (dashed) or both components (dotted) to contribute over the entire evolution from an initial separation of $2 R_{\mathrm{p}}$. The spread of the binaries across a range of timescales indicates either a range of ages for the binaries, a range of material strengths in terms of $\mu Q$, or a combination of both. 
to orbit expansion timescales in binary systems, finding an asynchronous secondary appears to indicate youth, implying that the separation has not expanded much and that these binaries formed in essentially their current configurations. The product $\mu Q$ could be larger by an order of magnitude if the binaries are as old as their collisional lifetimes, but then one must explain why the secondaries are not despun over the longer timescale.

The eccentric nature of the mutual orbits of $2004 \mathrm{DC}$ and $2003 \mathrm{YT}_{1}$ could be key to understanding why these systems stand out among the rest of the near-Earth binary population, perhaps through entering a tumbling state (Ćuk and Nesvornv́, 2010) similar to that of the saturnian satellite Hyperion (Wisdom et al., 1984). Ćuk and Nesvorný (2010) suggest that after the secondary is initially synchronized by tides, outward BYORP evolution can both provide an eccentricity to the mutual orbit and trigger episodes of chaotic rotation, breaking the synchronous lock of the secondary. Without a synchronous secondary, BYORP must shut down in these systems, but BYORP may have aided tides in expanding their mutual orbits. Because of the rapid timescales for orbit expansion associated with BYORP, the $\mu Q$ calculated assuming only tidal expansion would be artificially lowered due to ignoring the additional BYORP component. In other words, a smaller $\mu Q$ is needed for a rapid orbital expansion, but $\mu Q$ could be larger if tides are not the only orbit expansion mechanism. Therefore, these asynchronous, eccentric binaries with very small $\mu Q$ values may be indirect evidence in favor of the BYORP scenario. While this scenario would help explain the characteristics of the $2004 \mathrm{DC}$ and $2003 \mathrm{YT}_{1}$ systems, one must keep in mind that, contrary to Ćuk and Nesvorný (2010), McMahon and Scheeres (2010b) argue that the BYORP effect should circularize the mutual orbit as the separation increases. Clearly, further research on the dynamical evolution of binary asteroid systems due to BYORP is required.

In addition to BYORP, mass lofting could continuously expand the orbit (Harris et al., 2009; Fahnestock and Scheeres, 2009) while a close planetary encounter could impulsively expand the orbit and provide a moderate eccentricity (Chauvineau et al., 1995; Scheeres, 2007b). The short timescale for YORP spin-up could prevent some secondaries from synchronizing and may also be important in keeping the primary rotation rapid, hence fueling orbit expansion via mass lofting. If tidal despinning (and mass lofting) cannot regulate the spin of the primary, the primary could conceivably undergo another significant mass-shedding event producing a tertiary component leading to triple system, such as (153591) $2001 \mathrm{SN}_{263}$ (Nolan et al., 2008) and (136617) 1994 CC (Brozovic et al., 2010) in the near-Earth region, or to the ejection of one of the components from the system. It is likely a combination of these effects in the near-Earth region that produce the observed binary configurations as well as the seemingly unrealistic values of $\mu Q$ for some systems.

\subsection{Small main-belt binaries}

Small main-belt binaries, those whose primaries have radii of a few kilometers, represent a population that blurs the characteristics of the large main-belt binaries and the near-Earth binaries. The small main-belt binaries share heliocentric orbits in the same region as their much larger cousins, but have similar properties to the near-Earth binaries such as angular momentum content. In Table 3, the average $J / J^{\prime}$ is 0.41 , similar to the angular momentum required by the spin-fission binary formation mechanism, with $73 \%$ of the binaries having $0.3 \leq J / J^{\prime} \leq 0.5$. The minority of systems have angular momenta consistent both with sub-catastrophic collisions 
$\left(J / J^{\prime}<0.3\right)$ and giant impact or capture mechanisms $\left(J / J^{\prime}>0.5\right)$. If formed through spin-up, one would expect these systems to have $\mu Q$ values similar to the near-Earth binaries for similar ages. Because these systems are discovered with lightcurve photometry, the known population is limited to systems with size ratios of roughly $R_{\mathrm{S}} / R_{\mathrm{p}} \geq 0.2$, the reliable detection limit for mutual occultation/eclipse events (Pravec et al., 2006).

As seen with binaries like (90) Antiope, systems with nearly equal-mass components can tidally evolve to a fully synchronous end state very rapidly. If we ignore this type of system among the small main-belt binaries, where the spin periods have likely lengthened to equal the mutual orbit period, the average spin period of the primary components is $3.2 \mathrm{~h}$ and makes up $\sim 75 \%$ of the angular momentum of the system. This is similar to the 2.8 -h average period for primaries in near-Earth binary systems and another indication that a similar binary formation process is at work in the near-Earth region and among $\mathrm{km}$-scale main-belt asteroids. The YORP spin-doubling timescale for a parent body a few $\mathrm{km}$ in radius orbiting in the main belt is of order 100 Myr (Rubincam, 2000; Vokrouhlický and Čapek, 2002; Čapek and Vokrouhlický, 2004). Thus, in a few hundred million years, a small main-belt asteroid could spin up from a mundane period of 6 or $12 \mathrm{~h}$ to a period of $3 \mathrm{~h}$ or less producing a binary system with a rapidly spinning primary, where accounting for the time required to spin up the parent body does not drastically reduce the possible age of the resulting binary. The collisional lifetimes of the $\mathrm{km}$-scale secondaries, however, limit the ages of the small main-belt binaries to between roughly $100 \mathrm{Myr}$ and 1 Gyr (Bottke et al., 2005).

Assuming a Phobos-like $\mu Q$ of $10^{11} \mathrm{~N} \mathrm{~m}^{-2}$, the small main-belt binary systems in Fig. 5 cluster around an age of $100 \mathrm{Myr}$ with an order-of-magnitude scatter to both younger and older ages. If small main-belt binaries have existed instead for the maximum collisional lifetime of the secondaries of $1 \mathrm{Gyr}$, the median $\mu Q$ in Table 3 is $7 \times 10^{11} \mathrm{~N} \mathrm{~m}^{-2}$. For this value of the material strength, the secondaries in small main-belt binary systems are despun on 1-10 Myr timescales, quicker than the YORP timescale, and thus are expected to be synchronously rotating for systems that are 1 Gyr old, while the primaries are despun on timescales similar to the collisional lifetimes of the bodies or longer. Nearly equal-mass binaries can despin on $1 \mathrm{Myr}$ timescales assuming the median $\mu Q$ value.

The median $\mu Q$ we calculate is not only larger than that found for the large main-belt binaries, but it is reasonable for solid or somewhat fractured rock. Considering that the majority of small main-belt binaries appear to have formed via spin-up, unless the primaries are essentially solid cores left over from a parent body that shed its loose material to form a substantial secondary $\left(R_{\mathrm{s}} / R_{\mathrm{p}} \geq 0.2\right)$, the median $\mu Q$ for evolution over 1 Gyr seems like an overestimate. If the systems are much younger than their collisional lifetimes, for every order of magnitude younger the binary systems are, $\mu Q$ will also reduce by an order of magnitude. If small main-belt binaries are $100 \mathrm{Myr}$ old, the median $\mu Q$ value would be within a factor of two of Phobos or a typical large main-belt binary; if small main-belt binaries are $10 \mathrm{Myr}$ old, they would have a median $\mu Q$ an order of magnitude stronger than near-Earth binaries of the same age. An order-of-magnitude difference in $\mu Q$ between small main-belt binaries and near-Earth binaries could be a size effect since, in Goldreich and Sari's gravitational-aggregate model, the rigidity scales as the radius of the body (see Footnote 8). The average small main-belt primary in Table 3 is a factor of five larger than the average near-Earth primary in Table 2, and thus could account for the same factor in the rigidity of the bodies. If a size effect cannot account for the difference between the $\mu Q$ values found for 


\begin{tabular}{|c|c|c|c|c|c|c|c|c|}
\hline & Name & $R_{\mathrm{p}}(\mathrm{km})$ & $R_{\mathrm{s}} / R_{\mathrm{p}}$ & $q$ & $\rho\left(\mathrm{g} \mathrm{cm}^{-3}\right)$ & $a / R_{\mathrm{p}}$ & $J / J^{\prime}$ & $\mu Q\left(\mathrm{~N} \mathrm{~m}^{-2}\right)$ \\
\hline 809 & Lundia & 3.5 & 0.89 & 0.705 & 2.0 & 4.4 & 0.50 & $6.3 \times 10^{13}$ \\
\hline 854 & Frostia & 4.5 & 0.98 & 0.941 & 2.0 & 8.3 & 0.66 & $2.4 \times 10^{12}$ \\
\hline 1089 & Tama & 4.7 & 0.90 & 0.729 & 2.0 & 4.6 & 0.51 & $9.0 \times 10^{13}$ \\
\hline 1139 & Atami & 2.5 & 0.80 & 0.512 & 2.0 & 6.1 & 0.54 & $2.5 \times 10^{12}$ \\
\hline 1313 & Berna & 5.0 & 0.97 & 0.913 & 2.0 & 6.3 & 0.58 & $1.7 \times 10^{13}$ \\
\hline 1338 & Duponta & 3.7 & 0.24 & 0.014 & 2.0 & 4.0 & 0.25 & $2.0 \times 10^{12}$ \\
\hline 1453 & Fennia & 3.5 & 0.28 & 0.022 & 2.0 & 4.7 & 0.24 & $8.9 \times 10^{11}$ \\
\hline 1717 & Arlon & 4.5 & 0.60 & 0.216 & 2.0 & 14.8 & 0.67 & $9.5 \times 10^{9}$ \\
\hline 1830 & Pogson & 3.8 & 0.40 & 0.064 & 2.0 & 5.0 & 0.44 & $2.3 \times 10^{12}$ \\
\hline 2006 & Polonskaya & 2.8 & 0.23 & 0.012 & 2.0 & 4.2 & 0.31 & $7.3 \times 10^{11}$ \\
\hline 2044 & Wirt & 3.5 & 0.25 & 0.016 & 2.0 & 4.2 & 0.27 & $1.4 \times 10^{12}$ \\
\hline 2131 & Mayall & 3.7 & 0.30 & 0.027 & 2.0 & 4.8 & 0.39 & $1.1 \times 10^{12}$ \\
\hline 2478 & Tokai & 3.5 & 0.86 & 0.636 & 2.0 & 6.1 & 0.56 & $6.6 \times 10^{12}$ \\
\hline 2577 & Litva & 2.0 & 0.40 & 0.064 & 2.0 & 6.5 & 0.43 & $1.1 \times 10^{11}$ \\
\hline 2754 & Efimov & 3.0 & 0.20 & 0.008 & 2.0 & 3.5 & 0.38 & $1.7 \times 10^{12}$ \\
\hline 3073 & Kursk & 2.8 & 0.25 & 0.016 & 2.0 & 7.4 & 0.29 & $2.2 \times 10^{10}$ \\
\hline 3309 & Brorfelde & 2.5 & 0.26 & 0.018 & 2.0 & 4.1 & 0.38 & $9.7 \times 10^{11}$ \\
\hline 3673 & Levy & 3.2 & 0.27 & 0.020 & 2.0 & 4.6 & 0.36 & $8.7 \times 10^{11}$ \\
\hline 3703 & Volkonskaya & 1.4 & 0.40 & 0.064 & 2.0 & 5.0 & 0.37 & $3.1 \times 10^{11}$ \\
\hline 3749 & Balam & 2.7 & 0.40 & 0.064 & 2.0 & 6.2 & 0.43 & $2.9 \times 10^{11}$ \\
\hline 3782 & Celle & 3.0 & 0.43 & 0.080 & 2.0 & 6.6 & 0.38 & $2.9 \times 10^{11}$ \\
\hline 3868 & Mendoza & 4.2 & 0.22 & 0.011 & 2.0 & 4.9 & 0.34 & $5.0 \times 10^{11}$ \\
\hline 4029 & Bridges & 4.0 & 0.35 & 0.043 & 2.0 & 3.8 & 0.31 & $9.5 \times 10^{12}$ \\
\hline 4492 & Debussy & 5.5 & 0.93 & 0.804 & 2.0 & 6.4 & 0.59 & $1.6 \times 10^{13}$ \\
\hline 4607 & Seilandfarm & 4.5 & 0.30 & 0.027 & 2.0 & 5.9 & 0.28 & $4.5 \times 10^{11}$ \\
\hline 4786 & Titianina & 3.5 & 0.19 & 0.007 & 2.0 & 4.5 & 0.32 & $3.6 \times 10^{11}$ \\
\hline 4951 & Iwamoto & 2.0 & 0.88 & 0.681 & 2.0 & 16.8 & 0.90 & $3.2 \times 10^{9}$ \\
\hline 5407 & $1992 \mathrm{AX}$ & 2.0 & 0.20 & 0.008 & 2.0 & 3.3 & 0.36 & $1.1 \times 10^{12}$ \\
\hline 5477 & $1989 \mathrm{UH}_{2}$ & 1.5 & 0.40 & 0.064 & 2.0 & 5.0 & 0.40 & $3.4 \times 10^{11}$ \\
\hline 5481 & Kiuchi & 2.6 & 0.33 & 0.036 & 2.0 & 4.5 & 0.31 & $1.2 \times 10^{12}$ \\
\hline 5905 & Johnson & 1.8 & 0.38 & 0.055 & 2.0 & 4.6 & 0.32 & $7.2 \times 10^{11}$ \\
\hline 6084 & Bascom & 2.9 & 0.37 & 0.051 & 2.0 & 7.4 & 0.42 & $8.2 \times 10^{10}$ \\
\hline 6244 & Okamoto & 2.6 & 0.25 & 0.016 & 2.0 & 4.4 & 0.34 & $6.1 \times 10^{11}$ \\
\hline 6265 & $1985 \mathrm{TW}_{3}$ & 3.0 & 0.24 & 0.014 & 2.0 & 3.7 & 0.35 & $2.0 \times 10^{12}$ \\
\hline 6708 & Bobbievaile & 2.9 & 0.60 & 0.216 & 2.0 & 5.3 & 0.41 & $3.3 \times 10^{12}$ \\
\hline 7088 & Ishtar & 0.6 & 0.42 & 0.074 & 2.0 & 4.5 & 0.43 & $1.3 \times 10^{11}$ \\
\hline 7255 & Huntress & 2.7 & 0.21 & 0.009 & 2.0 & 3.5 & 0.38 & $1.7 \times 10^{12}$ \\
\hline 7369 & Gavrilin & 2.4 & 0.70 & 0.343 & 2.0 & 8.7 & 0.55 & $1.5 \times 10^{11}$ \\
\hline 8116 & Jeanperrin & 2.3 & 0.40 & 0.064 & 2.0 & 6.5 & 0.37 & $1.5 \times 10^{11}$ \\
\hline 8373 & Stephengould & 3.0 & 0.40 & 0.064 & 2.0 & 6.3 & 0.32 & $3.1 \times 10^{11}$ \\
\hline 9260 & Edwardolson & 1.9 & 0.27 & 0.020 & 2.0 & 4.0 & 0.32 & $7.3 \times 10^{11}$ \\
\hline 9617 & Grahamchapman & 2.5 & 0.27 & 0.020 & 2.0 & 4.2 & 0.42 & $8.7 \times 10^{11}$ \\
\hline 10208 & $1997 \mathrm{QN}_{1}$ & 1.7 & 0.46 & 0.097 & 2.0 & 9.1 & 0.47 & $1.4 \times 10^{10}$ \\
\hline 11264 & Claudiomaccone & 2.1 & 0.40 & 0.064 & 2.0 & 3.6 & 0.36 & $5.5 \times 10^{12}$ \\
\hline 15268 & Wendelinefroger & 2.1 & 0.30 & 0.027 & 2.0 & 5.0 & 0.41 & $2.8 \times 10^{11}$ \\
\hline 16635 & $1993 \mathrm{QO}$ & 1.9 & 0.35 & 0.043 & 2.0 & 6.0 & 0.48 & $1.1 \times 10^{11}$ \\
\hline 17260 & $2000 \mathrm{JQ}_{58}$ & 1.6 & 0.26 & 0.018 & 2.0 & 3.5 & 0.31 & $1.0 \times 10^{12}$ \\
\hline 26471 & $2000 \mathrm{AS}_{152}$ & 3.2 & 0.36 & 0.047 & 2.0 & 6.8 & 0.42 & $1.5 \times 10^{11}$ \\
\hline 32008 & $2000 \mathrm{HM}_{53}$ & 1.7 & 0.50 & 0.125 & 2.0 & 7.1 & 0.50 & $8.8 \times 10^{10}$ \\
\hline 34706 & $2001 \mathrm{OP}_{83}$ & 1.6 & 0.28 & 0.022 & 2.0 & 4.4 & 0.38 & $2.9 \times 10^{11}$ \\
\hline 76818 & $2000 \mathrm{RG}_{79}$ & 1.4 & 0.35 & 0.043 & 2.0 & 3.5 & 0.34 & $2.3 \times 10^{12}$ \\
\hline
\end{tabular}

Table 3: Physical properties of small main-belt binary asteroids with an average primary radius of 3 $\mathrm{km}$. Densities are estimated as $2 \mathrm{~g} \mathrm{~cm}^{-3}$ (see Pravec and Harris, 2007, for details) in all cases. The typical $J / J^{\prime}$ roughly satisfies the condition for binary formation via spin-up of a single parent body as with the near-Earth binaries. Values of $\mu Q$ correspond to tides raised on the primary acting over the 1 Gyr collisional lifetime of the secondaries. Accounting for tides raised on the secondary would increase $\mu Q$ by no more than a factor of two. Data are taken from the Ondrejov Asteroid Photometry Project binary asteroid parameters table (http://www.asu.cas.cz/ asteroid/binastdata.htm, 2010 April 8 release). 
small main-belt and near-Earth binaries of similar ages, then this discrepancy could indicate that the $\mu Q$ values found for near-Earth binaries are artificially lowered due to the presence (and our ignorance) of other orbit expansion mechanisms.

Small main-belt binaries, if formed via spin-up, should form on a rather consistent basis over the last billion years, yet we find them to clump together at a similar age in Figure 5. For the

median value of $\mu Q$, this would be $1 \mathrm{Gyr}$, but the median $\mu Q$ value seems at odds with the weaker structure one expects for a binary formed via spin-up. Lowering the age of the population to reduce the median $\mu Q$ of the small main-belt binaries would make it seem that the small main-belt binary population is missing an "older" component. For a Phobos-like $\mu Q$ of $10^{11} \mathrm{~N} \mathrm{~m}^{-2}$, binaries with an age of about 1 Gyr are under-represented if binaries have formed on a consistent basis, and the smaller the median $\mu Q$ of the binaries, the younger the observed population and also the larger the proportion of "missing" binaries. If detection bias against longer orbit periods in photometric surveys (Pravec et al., 2006) is not the sole cause of the relative dearth of wider binaries that would represent an older population for the same $\mu Q$ value, perhaps this is evidence that older (100 Myr to 1 Gyr old) binaries have been destroyed via collisions or that another mechanism exists in the main belt that is capable of destroying binaries. BYORP is capable of contracting the mutual orbits of binary systems, leading either to the coalescence of the components or a masking of the true age of the system by hindering tidal expansion. Unchecked orbit expansion by BYORP could conceivably lead to the loss of the secondary, though complete dissociation of the components via BYORP is argued against by Ćuk and Nesvorný (2010). The YORP effect is also capable of collapsing binaries by removing angular momentum from the system until a fully synchronous orbit no longer exists (Taylor, 2009). Continued YORP spin-up and repeated mass-shedding events could conceivably produce a tertiary component in less than the YORP spin-doubling timescale of $100 \mathrm{Myr}$. If the triple system is unstable, one body could be ejected such that the binaries we observe are simply the most recent incarnation of the system. Note, however, that while the YORP timescale is shorter than the tidal despinning timescale for the primary assuming the median $\mu Q$ value, if small main-belt binaries are $10 \mathrm{Myr}$ old instead of 1 Gyr old, $\mu Q$ is reduced, and tidal despinning should then overcome YORP spin-up, preventing further mass-shedding events and slowing down the primary's rotation from the breakup rate.

\subsection{Other considerations}

Several factors can affect the calculation of $\mu Q$ in addition to the major source of error, the assumed age of the binary $\Delta t$. These include measurement errors in the (1) sizes and densities of the components or (2) the current separation $a / R_{\mathrm{p}},(3)$ the assumption of an initial separation, and (4) neglect of higher-order terms in the tidal potential at small separations. These considerations are discussed in more detail in Taylor and Margot (2010) and are summarized here:

1. The calculation of $\mu Q$ scales as $\rho_{\mathrm{p}}^{5 / 2} R_{\mathrm{p}}^{2} q(1+q)^{1 / 2} \propto \rho_{\mathrm{p}}^{11 / 6} q(1+q)^{1 / 2}$ for a binary with a known system mass such that underestimates (overestimates) in any of these parameters by some percentage lead to underestimates (overestimates) of $\mu Q$ by a larger percentage. The standard assumption of equal component densities could be troublesome, as for (66391) 1999 $\mathrm{KW}_{4}$, where the difference in component densities (Ostro et al., 2006) results in a $43 \%$ error in $q$ that can skew $\mu Q$ by a similar amount. 


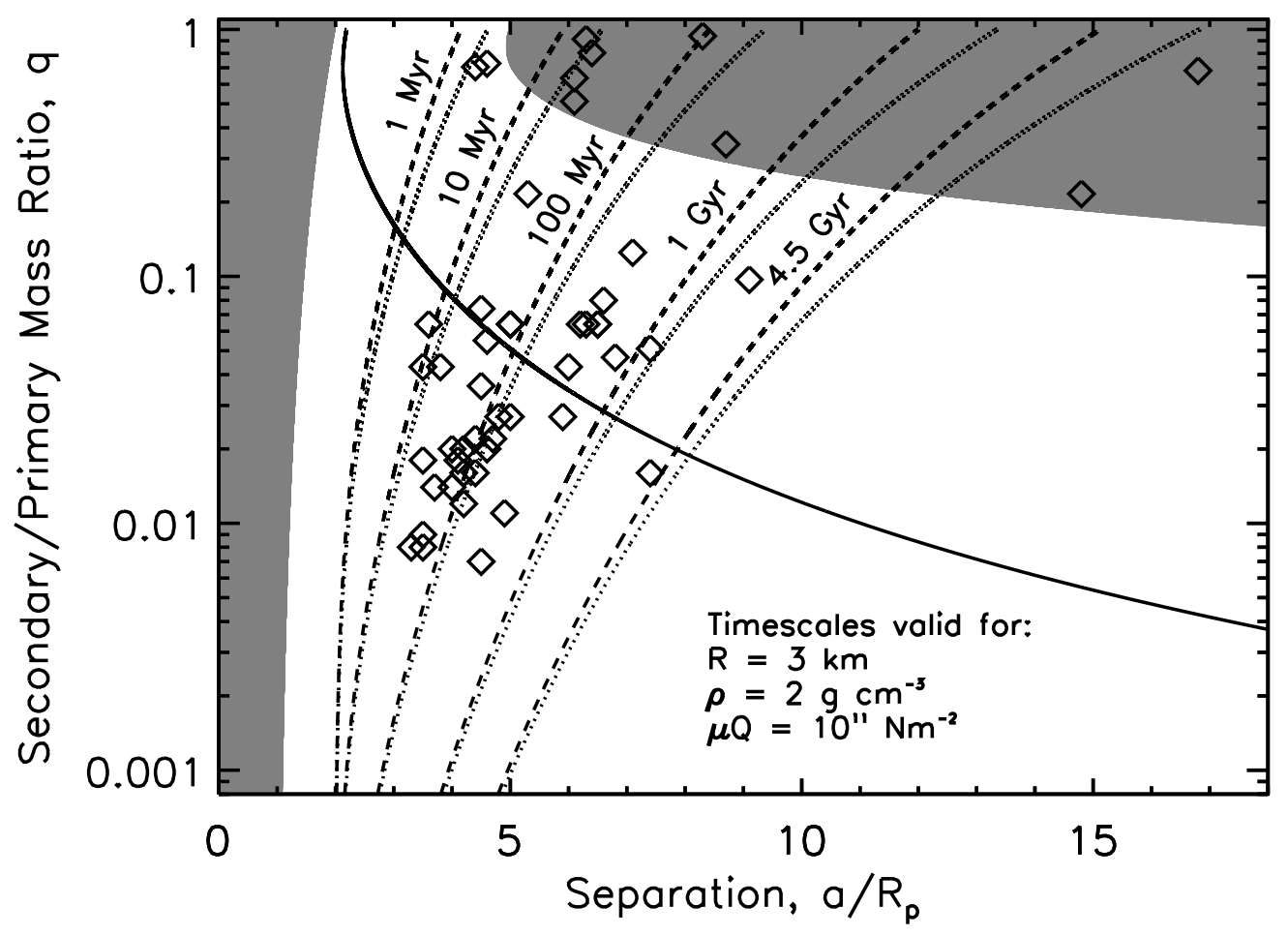

Fig. 5. Mass ratio $q$ and primary-secondary separation $a / R_{\mathrm{p}}$ for small main-belt binaries along with the angular momentum limit for $J / J^{\prime}=0.5$. The solid curve is the stability limit. Tidal evolution timescales are plotted assuming $\rho_{\mathrm{p}, \mathrm{s}}=2 \mathrm{~g} \mathrm{~cm}^{-3}, R_{\mathrm{p}}=3 \mathrm{~km}$, and $\mu_{\mathrm{p}} Q_{\mathrm{p}}=\mu_{\mathrm{s}} Q_{\mathrm{s}}=10^{11} \mathrm{~N} \mathrm{~m}^{-2}$ and allowing tides raised on the primary only (dashed) or both components (dotted) to contribute over the entire evolution from an initial separation of $2 R_{\mathrm{p}}$. The binaries tend to cluster near size ratios of $0.2(q \sim 0.01)$, the lower limit for reliable mutual event detection by photometry, and an age of 100 Myr for the assumed $\mu Q$ value. 
2. An error in the current separation $a / R_{\mathrm{p}}$ of $10 \%$ can cause a factor of two error in the calculated value of $\mu Q$. Typical separation uncertainties are estimated as $10-15 \%$ by the Ondrejov Asteroid Photometry Project.

3. Assuming an initial separation of $2 R_{\mathrm{p}}$ does not affect the calculated $\mu Q$ by more than a factor of two unless the true initial separation is within $10 \%$ of the current (final) separation. We note in Table 1 that the (617) Patroclus binary system must have formed at a wider separation than the assumed value of $2 R_{\mathrm{p}}$, but only if the true initial separation were greater than $12 R_{\mathrm{p}}$ would the estimate of $\mu Q$ increase by more than a factor of two.

4. Allowing for higher-order terms in tidal potential due to the proximity of the components can increase $\mu Q$ by $20-30 \%$ for separations near $2 R_{\mathrm{p}}$, but the contribution of extra terms quickly falls off to $5 \%$ for tidal evolution to $5 R_{\mathrm{p}}$ and to $1 \%$ at $10 R_{\mathrm{p}}$.

None of the above considerations should affect the calculation of $\mu Q$ by more than a factor of two or so on their own; only a severe measurement error or several of these factors working in concert would affect an order-of-magnitude calculation of the material properties of a binary asteroid system.

Also of concern are solar tides, whose presence are felt through additional tidal distortions raised on each component. The ratio of the tidal amplitudes raised on the primary due to the Sun and the secondary scales as $\left(M_{\odot} / M_{\mathrm{S}}\right)\left(a / a_{\odot}\right)^{3}$, where $M_{\odot}$ is the mass of the Sun and $a_{\odot}$ is the heliocentric semimajor axis of the binary system. For a typical near-Earth binary, $M_{\odot} / M_{\mathrm{S}} \sim 10^{19}$ and $a / a_{\odot} \sim 10^{-8}$ such that the ratio of tidal amplitudes is $10^{-5}$ rendering solar tides negligible unless the binary has a shallow perihelion of a few tenths of an astronomical unit (Scheeres et al., 2006; (Scheeres, 2007b). Despite their small masses, the secondaries in near-Earth binary systems are so close to their primaries that they easily raise the dominant tide. For a typical large main-belt binary $M_{\odot} / M_{\mathrm{s}} \sim 10^{15}$ and $a / a_{\odot} \sim 10^{-6}$ such that the ratio of tidal amplitudes is $10^{-3}$, which is still negligible in the scope of this discussion since large main-belt asteroids cannot have close flybys of the Sun. The wider separation of main-belt binary components weakens the tide raised by the secondary, but it still dominates over the solar tide. Typical small main-belt binaries have $M_{\odot} / M_{\mathrm{S}} \sim 10^{17}$ and $a / a_{\odot} \sim 10^{-8}$ such that the ratio of the tidal amplitudes is $10^{-7}$ making them the least affected by solar tides. This is due to having larger secondaries than the near-Earth binaries and having smaller component separations than the large main-belt binaries. Since the mass of the secondary is typically at least two orders of magnitude less than the primary, solar tides on the secondary are also negligible.

Thus far, we have only considered binary components with equal densities. Applying a density ratio of $0.5 \leq \rho_{\mathrm{p}} / \rho_{\mathrm{s}} \leq 1.5$ in Eq. 7 has little effect on the locations of the fully synchronous orbits (see Fig. 6) as the density ratio appears only as a pre-factor of the mass ratio $q$, which tends to be small. For typical binaries with $J / J^{\prime}=0.2$ or 0.4 and $q<0.1$, the effect of a density disparity on $a_{\text {sync }} / R_{\mathrm{p}}$ is imperceptible. For $J / J^{\prime}=0.5$ and $q=1$, the effect is almost $10 \%$, similar to the measurement uncertainty on the separation, though one would expect similar-size bodies in a binary system to have similar densities. Of course, this has assumed that introducing a density disparity is the only alteration to Eq. 7. but changing the density ratio will necessarily change the mass ratio according to the definition of $q$ (that in turn affects the calculation of $\mu Q$ as in point $\# 1$ above) and change the angular momentum $J / J^{\prime}$ as well. For $(66391) 1999 \mathrm{KW}_{4}, \rho_{\mathrm{p}} / \rho_{\mathrm{s}}=0.7$, which increases the mass ratio $q$ by $43 \%$ and increases $J / J^{\prime}$ by $5 \%$ to support the rotation and 
orbital motion of a more massive secondary. The stable fully synchronous end state shifts from $\sim 120 R_{\mathrm{p}}$ to $\sim 70 R_{\mathrm{p}}$ due to the combined effects of the larger mass ratio (that shifts $a_{\text {sync }} / R_{\mathrm{p}}$ inward by nearly a factor of twd 9 ) and the increased angular momentum of the system (that shifts $a_{\text {sync }} / R_{\mathrm{p}}$ outward by $10 \%$ ). While this change in position is significant, the $1999 \mathrm{KW}_{4}$ system, currently at $4 R_{\mathrm{p}}$, will never attain the stable fully synchronous end state via tidal evolution due to the insurmountable orbit-expansion timescale at wide separations compared to the dynamical lifetime of a near-Earth asteroid and because the stable fully synchronous end state lies beyond the Hill sphere of the primary. It is clearly the effect of the density disparity on the mass ratio $q$, capable of changing $q$ by a factor of two for $0.5 \leq \rho_{\mathrm{p}} / \rho_{\mathrm{s}} \leq 1.5$, that is more important to determining the locations of the stable fully synchronous tidal end state and calculating $\mu Q$ than the density ratio as it appears directly in Eq. 7 .

We have also only considered spherical bodies with uniform densities thus far. In Section 4 , we noted how determining the fully synchronous orbits about a nonspherical body requires a more complicated treatment of the mutual gravitational potential and the synchronous rotation rate necessary for relative equilibrium than presented here. Making a body ellipsoidal also increases $\alpha$ as it requires more angular momentum to rotate an ellipsoid at a given rate about its axis of maximum moment of inertia than it does a sphere; on the other hand, concentrating mass toward the center of the body decreases $\alpha$. For a typical binary system with disparate masses, the spin of the primary dominates the angular momentum of the system such that a change in $\alpha_{\mathrm{p}}$ by some percentage can change the angular momentum by a similar, but lesser, percentage and affect the locations of the fully synchronous orbits in Fig. 1 accordingly. For systems with disparate masses, the spin of the secondary contributes to the total angular momentum at the $1 \%$ level or less such that the exact value of $\alpha_{\mathrm{s}}$ is unimportant. As with the density ratio, the $\alpha_{\mathrm{s}} / \alpha_{\mathrm{p}}$ ratio only appears as a pre-factor of the mass ratio $q$ in Eq. 7, and thus is insignificant unless $q \sim 1$; however, equal-mass binaries are dominated by the orbital rather than spin angular momentum when residing in a stable fully synchronous orbit. Therefore, only the value of $\alpha_{\mathrm{p}}$ is significant and only then for systems with components of unequal mass. Take, for example, a typical near-Earth binary where $80 \%$ of the angular momentum is in the spin of the primary, then allowing $\alpha_{\mathrm{p}}$ to vary by $25 \%$ about the nominal value of 0.4 would in turn let the angular momentum of the system vary by $20 \%$ or between $J / J^{\prime}=0.32$ and 0.48 assuming the nominal angular momentum of $J / J^{\prime}=0.4$ for a binary formed by spin-up. While such a range in $J / J^{\prime}$ opens a wide swath in Fig. 1, the stable fully synchronous orbit will typically be far from the primary, ranging from tens of primary radii away for $q=0.1$ to hundreds or thousands of primary radii (or more) as $q$ decreases, separations that are comparable to or beyond the Hill sphere of the primary.

\section{Discussion}

We have derived and plotted the locations of the fully synchronous tidal end states for all mass ratios and angular momenta and find that only binary asteroid systems with nearly equal-mass components such as (90) Antiope and (69230) Hermes reside in these end states with all other

\footnotetext{
${ }^{9}$ In Figs. 1 and 6, for mass ratios of $q<0.5$, the location of the stable fully synchronous orbit rapidly recedes such that a small change in mass ratio corresponds to a drastic change in the orbital separation.
} 


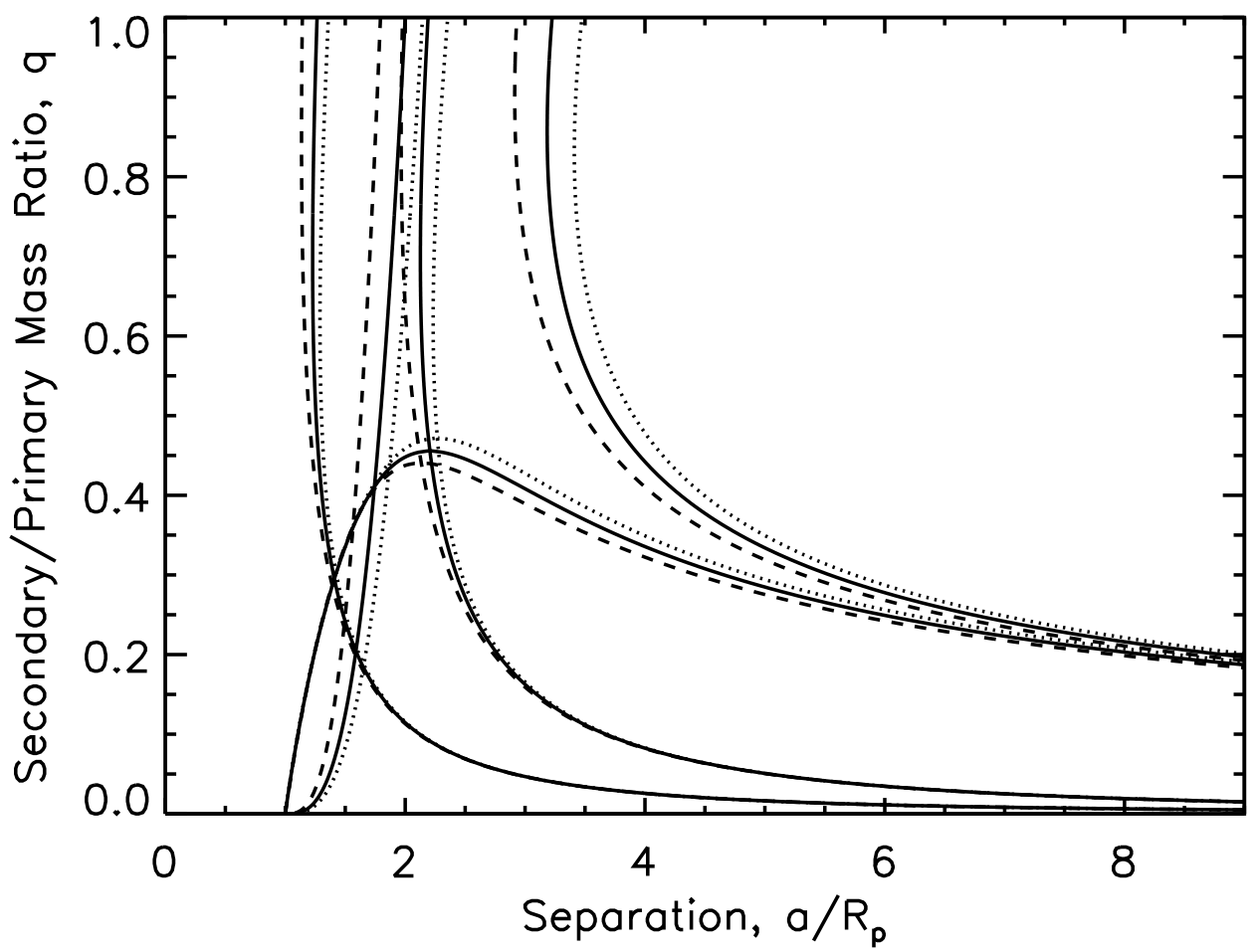

Fig. 6. Fully synchronous orbits (Eq. 7) for binary systems with scaled angular momentum $J / J^{\prime}=$ 0.4 along with the contact limit (Eq. 44), angular momentum limit (Eq. 5), stability limit (Eq. 10), and $E=0$ energy limit (Eq. 15) for density ratios of $\rho_{\mathrm{p}} / \rho_{\mathrm{s}}=0.5$ (dashed), $\rho_{\mathrm{p}} / \rho_{\mathrm{s}}=1$ (solid), and $\rho_{\mathrm{p}} / \rho_{\mathrm{s}}=1.5$ (dotted). A less dense primary shifts all curves inward, while a higher-density primary shifts the curves outward, though the effect is insignificant for mass ratios less than a few tenths. Spherical shapes with $\alpha_{\mathrm{p}, \mathrm{s}}=0.4$ are assumed in all cases. 
systems with smaller mass ratios undergoing a lengthy tidal evolution process. Though (90) Antiope and (69230) Hermes are excellent examples of the stable fully synchronous tidal end state, these systems can evolve to their current states so rapidly for reasonable values of the product of rigidity $\mu$ and tidal dissipation function $Q$ that their material properties are difficult to constrain. Relying instead upon the binary systems with smaller mass ratios, we find among 100-km-scale main-belt binaries values of the material strength $\mu Q$ that, for tidal evolution over the age of the Solar System, are consistent with solid or fractured rock as one might expect for binaries created via sub-catastrophic collisions. Binaries formed in the near-Earth region via spin-up that have evolved over the typical $10 \mathrm{Myr}$ dynamical lifetime require material strengths orders of magnitude smaller than their large main-belt counterparts as one might expect for gravitational aggregates of material compared to essentially monolithic bodies. If near-Earth binaries formed in the main belt prior to injection to the near-Earth region, the time available for tidal evolution can lengthen and $\mu Q$ can increase in a similar manner. The discovery of many small main-belt binaries with rapidly spinning primaries and angular momenta similar to the near-Earth binaries lends some credence to the idea that near-Earth binaries, or some fraction of near-Earth binaries, could form in the main belt prior to delivery to the near-Earth region and have binary ages that are limited by collisional lifetimes rather than the dynamical lifetime in the near-Earth region. An older age for some near-Earth binaries, i.e., $100 \mathrm{Myr}$ versus $10 \mathrm{Myr}$, would help reconcile some especially small values of $\mu Q$ with the model of gravitational-aggregate structure of Goldreich and Sari (2009). If small main-belt binaries are up to 1 Gyr old, the maximum collisional lifetime of their secondaries, their material strengths are more consistent with solid rock than gravitational aggregates. Younger ages similar to the near-Earth binaries would reduce $\mu Q$, but leave an older binary population unaccounted for either because of detection bias, collisional destruction, or another destruction mechanism. Smaller $\mu Q$ values for the small main-belt binaries would be more consistent with the gravitational-aggregate structure implied by the likely formation mechanism of YORP spin-up and would allow tidal spin-down to overcome continued YORP spin-up of the primary, which could account for the slightly slower rotation rates of small main-belt primaries compared to near-Earth primaries.

Beyond the question of age, our ignorance of the inherent $Q$ of gravitational aggregates and whether they can be described in such idealized terms are significant caveats in this approach to understanding the material properties of asteroids. Especially in the near-Earth region, the assumption that tides are the dominant method of mutual-orbit expansion may also be tenuous. The binary YORP effect, mass lofting, and close planetary encounters could conspire to also expand or contract the mutual orbits, either assisting or hindering the expansion of the mutual orbit by tides. A combination of effects altering the semimajor axis would skew the resulting $\mu Q$ when considering tides alone; multiple methods of orbital expansion working in concert would give the illusion of a small $\mu Q$ value rather than the true material strength of the body. It is difficult to disentangle different orbit-expansion mechanisms on top of not knowing the precise age of a binary system. If we take tides to be the dominant orbit-expansion mechanism, then the assumption of the binary age $\Delta t$ is easily the largest source of error in the calculation of $\mu Q$ for a binary system causing an order-of-magnitude error in $\mu Q$ for an order-of-magnitude error in $\Delta t$. Other sources of error, those summarized in Section 5.6 and also from ignoring tides raised on the secondary, will typically cause errors of less than a factor of two.

In our examination of tidal evolution, a few observations have hinted at the presence of a 
mechanism (or mechanisms) in addition to tides that evolves the semimajor axis of binary asteroid systems. Following Goldreich and Sari (2009), using their model for gravitational-aggregate structure and assuming the actual strength of the BYORP torque on the mutual orbit is $10^{-3}$ times its maximum possible value 10 it is found that BYORP should dominate the semimajor-axis evolution of all near-Earth binary systems with synchronous secondaries. For small main-belt binaries with unequal-mass components, BYORP and tides could contribute comparably to the semimajor-axis evolution, and even for large main-belt binaries, BYORP could be important under certain circumstances. Among the near-Earth binaries, the systems most susceptible to BYORP at some point in their evolution are $2004 \mathrm{DC}$ and $2003 \mathrm{YT}_{1}$, precisely the systems whose asynchronous secondaries, eccentric mutual orbits, and small and worrisome $\mu Q$ values led us to suspect a BYORP component to their evolution in Section 5.4. Large main-belt binary systems with mass ratios above $\sim 0.01$ are dominated by tides at their current separations. Large main-belt binaries with the smallest size ratios are more susceptible to BYORP, and we find that those that plot furthest to the right of the 4.5 Gyr curve in Fig. 3 and, hence, have the smallest calculated $\mu Q$ values: (45) Eugenia, (130) Elektra, and (702) Alauda, are most likely to be dominated by the BYORP effect at this time, which would lead to artificially low $\mu Q$ values compared to the rest of the binaries in Table 1 .

In the past, at smaller separations, these low mass-ratio systems among the large main-belt binaries would have been tide-dominated, which would allow for synchronization of the secondary with a rough transition to BYORP-dominated semimajor-axis evolution occurring around $6 R_{\mathrm{p}}$ if the strength of the BYORP effect is correctly estimated. Subsequent expansion by BYORP and tides would then produce the currently observed systems where assuming tide-dominated evolution finds a smaller value for $\mu Q$ than is necessarily true. This fact is related to point \#3 in Section 5.6. the calculation of $\mu Q$ is most sensitive to the evolution within $10 \%$ of the current separation such that if BYORP is a significant contributor to the semimajor-axis expansion in this range, then $\mu Q$ will be skewed to a lower value than is appropriate for the material. If the strength of the BYORP torque is $10^{-5}$ times its maximum possible value instead, the majority of near-Earth binaries and the three large main-belt systems mentioned would remain BYORP-dominated in their current configurations. The small main-belt binaries would be tide-dominated currently making it more difficult for destruction by BYORP to account for the apparent lack of older systems. If the strength of the BYORP torque is reduced even further to $10^{-7}$ times its maximum possible value, then only 2004 DC and $2003 \mathrm{YT}_{1}$ (and possibly $2000 \mathrm{DP}_{107}$ ) would remain BYORP-dominated. Given that BYORP could have a significant impact on many of the binary systems we have considered, perhaps the best example for the calculation of $\mu Q$ is (22) Kalliope, which has a small enough mass ratio that the system is still tidally evolving, unlike (90) Antiope or (69230) Hermes, yet not so small that BYORP could be an issue. Given the slew of sources of uncertainty in the calculation of material properties via tidal evolution, Kalliope's $\mu Q$ should lie within a factor of a few of $3.3 \times 10^{12} \mathrm{~N} \mathrm{~m}^{-2}$ based on a 4.5 Gyr evolution, which implies stronger material properties than Phobos, but is reasonable for a monolithic or fractured asteroid.

For a tide-dominated binary system, assuming an age equal to the age of the Solar System provides a confident order-of-magnitude upper bound on the value of $\mu Q$. One way to improve

${ }^{10} \mathrm{~A}$ similar factor for the YORP torque was found to be $4 \times 10^{-4}$ for asteroid (54509) YORP (formerly $2000 \mathrm{PH}_{5}$ ) by Tavlor et al. (2007) and Lowry et al. (2007). 
on this upper bound is to limit the evolution time by using collisional lifetimes (as discussed for near-Earth and small main-belt binaries) or by finding binaries in collisional families. If we assume the binary was formed as the result of the catastrophic disruption of a parent body that produced a family of asteroids (i.e., EEBs, escaping ejecta binaries, as described by Durda et al. (2004), then the binary evolves only over the age of the family. Changing the evolution time by some factor will affect the value of $\mu Q$ in the same manner. Of the main-belt binaries listed in Tables 1 and 3 , about a dozen (19\%) are believed to be part of the collisional families determined by Zappalà et al. (1995), smaller than the value of roughly 30\% found among all asteroids (Marzari et al., 1999; Bendjoya and Zappalà, 2002). Those families the binaries are associated with that have age estimates, i.e., Flora (Nesvornv́ et al., 2002a), Themis (Marzari et al., 1995, 1999; Nesvornv́ et al., 2005), and Eos (Vokrouhlický et al., 2006), are believed to be of order 1 Gyr old so that limiting the ages of the binaries to the age of the families rather than the age of the Solar System does not change the upper bound of $\mu Q$ by more than a factor of a few. If one could instead identify binary systems among much younger groups, such as the Karin or Veritas clusters, both of which are younger than $10 \mathrm{Myr}$ (Nesvorný et al., 2002b, 2003), the ages of the binaries, and thus their $\mu Q$ values, would be far better constrained.

\section{Acknowledgments}

The authors wish to thank Jean-Marc Petit and an anonymous referee for their detailed reviews that improved the clarity and quality of the manuscript. This work was supported by NASA Planetary Astronomy grants NNG04GN31G and NNX07AK68G/NNX09AQ68G to Jean-Luc Margot.

\section{References}

Bellerose, J., Scheeres, D.J., 2008. Energy and stability in the full two-body problem. Cel. Mech. Dynam. Astron. 100, 63-91.

Belton, M.J.S., et al., 1996. The discovery and orbit of 1993 (243)1 Dactyl. Icarus 120, 185-199.

Bendjoya, P., Zappalà, V., 2002. Asteroid family identification. In: Bottke, W.F., Cellino, A., Paollichi, P., Binzel, R.P. (Eds.), Asteroids III. Univ. of Arizona Press, Tucson, pp. 613-618.

Bottke, W.F., Melosh, H.J., 1996a. The formation of asteroid satellites and doublet craters by planetary tidal forces. Nature 381, 51-53.

Bottke, W.F., Melosh, H.J., 1996b. Binary asteroids and the formation of doublet craters. Icarus 124, 372-391.

Bottke, W.F., Vokrouhlický, D., Rubincam, D.P., Brož, M., 2002. The effect of Yarkovsky thermal forces on the dynamical evolution of asteroids and meteoroids. In: Bottke, W.F., Cellino, A., Paolicchi, P., Binzel, R.P. (Eds.), Asteroids III. Univ. of Arizona Press, Tucson, pp. 395-408.

Bottke, W.F., et al., 2005. Linking the collisional history of the main asteroid belt to its dynamical excitation and depletion. Icarus 179, 63-94. 
Bottke, W.F., Vokrouhlický, D., Rubincam, D.P., Nesvorný, D., 2006. The Yarkovsky and YORP effects: Implications for asteroid dynamics. Annu. Rev. Earth Planet. Sci. 34, 157-191.

Britt, D.T., Yeomans, D., Housen, K., Consolmagno, G., 2002. Asteroid density, porosity, and structure. In: Bottke, W.F., Cellino, A., Paolicchi, P., Binzel, R.P. (Eds.), Asteroids III. Univ. of Arizona Press, Tucson, pp. 485-500.

Brozovic, M., et al., 2010. Radar images and shape model of a triple Asteroid (136617) 1994 CC. Bull. Am. Astron. Soc. 42, (Abstract \#57.02).

Burns, J.A., Safronov, V.S., 1973. Asteroid nutation angles. Mon. Not. R. Astron. Soc. 165, 403-411.

Canup, R.M., 2005. A giant impact origin of Pluto-Charon. Science 307, 546-550.

Čapek, D., Vokrouhlický, D., 2004. The YORP effect with finite thermal conductivity. Icarus 172, $526-536$.

Chapman, C.R., et al., 1995. Discovery and physical properties of Dactyl a satellite of Asteroid 243 Ida. Nature 374, 783-785.

Chauvineau, B., Farinella, P., Harris, A.W., 1995. The evolution of Earth-approaching binary asteroids: A Monte Carlo dynamical model. Icarus 115, 36-46.

Christy, J.W., Harrington, R.S., 1978. The satellite of Pluto. Astron. J. 83, 1005-1008.

Counselman, C.C., III, 1973. Outcomes of tidal evolution. Astroph. J. 180, 307-316.

Ćuk, M., Burns, J.A., 2005. Effects of thermal radiation on the dynamics of binary NEAs. Icarus 176, 418-431.

Ćuk, M., Nesvorný, D., 2010. Orbital evolution of small binary asteroids. Icarus 207, 732-743.

Darwin, G.H., 1887. On figures of equilibrium of rotating masses of fluids. Philos. Trans. 178, $379-430$.

Descamps, P., Marchis, F., 2008. Angular momentum of binary asteroids: Implications for their possible origin. Icarus 193, 74-84.

Descamps, P., et al., 2007. Figure of the double Asteroid 90 Antiope from adaptive optics and lightcurve observations. Icarus 187, 482-499.

Durda, D.D., et al., 2004. The formation of asteroid satellites in large impacts: Results from numerical simulations. Icarus 170, 243-257.

Dziewonski, A.M., Anderson, D.L., 1981. Preliminary reference Earth model. Phys. Earth Planet. Int. 25, 297-356.

Efroimsky, M., Williams, J.G., 2009. Tidal torques: A critical review of some techniques. Cel. Mech. Dynam. Astron. 104, 257-289. 
Fahnestock, E.G., Scheeres, D.J., 2009. Binary asteroid orbit expansion due to continued YORP spin-up of the primary and primary surface particle motion. Icarus 201, 135-152.

Farinella, P., 1992. Evolution of Earth-crossing binary asteroids due to gravitational encounters with the Earth. Icarus 96, 284-285.

Gladman, B.J., et al., 1997. Dynamical lifetimes of objects injected into asteroid belt resonances. Science 277, 197-201.

Gladman, B., Michel, P., Froeschlé, C., 2000. The near-Earth object population. Icarus 146, 176-189.

Goldreich, P., 1963. On the eccentricity of satellite orbits in the Solar System. Mon. Not. R. Astron. Soc. 126, 257-268.

Goldreich, P., Soter, S., 1966. Q in the Solar System. Icarus 5, 375-389.

Goldreich, P., Sari, R., 2009. Tidal evolution of rubble piles. Astroph. J. 691, 54-60.

Harris, A.W., 1994. Tumbling asteroids. Icarus 107, 209-211.

Harris, A.W., Ward, W.R., 1982. Dynamical constraints on the formation and evolution of planetary bodies. Annu. Rev. Earth Planet. Sci. 10, 61-108.

Harris, A.W., Fahnestock, E.G., Pravec, P., 2009. On the shapes and spins of "rubble pile" asteroids. Icarus 199, 310-318.

He, H., Ahrens, T.J., 1994. Mechanical properties of shock-damaged rocks. J. Rock Mech. Min. Sci. Geomech. 31, 525-533.

Holsapple, K.A., 2010. On YORP-induced spin deformations of asteroids. Icarus 205, 430-442.

Holsapple, K.A., Michel, P., 2008. Tidal disruptions. II. A continuum theory for solid bodies with strength, with applications to the Solar System. Icarus 193, 283-301.

Hut, P., 1980. Stability of tidal equilibrium. Astron. Astroph. 92, 167-170.

Kaasalainen, M., Ďurech, J., Warner, B.D., Krugly, Y.N., Gaftonyuk, N.M., 2007. Acceleration of the rotation of Asteroid 1862 Apollo by radiation torques. Nature 446, 420-422.

Levrard, B., Winisdoerffer, C., Chabrier, G., 2009. Falling transiting extrasolar giant planets. Astroph. J. 692, L9-L13.

Lowry, S.C., et al., 2007. Direct detection of the asteroidal YORP effect. Science 316, 272-274.

Marchis, F., et al., 2006. A low density of $0.8 \mathrm{~g} \mathrm{~cm}^{-3}$ for the Trojan binary Asteroid 617 Patroclus. Nature 439, 565-567.

Marchis, F., et al., 2008a. Main belt binary asteroidal systems with eccentric mutual orbits. Icarus 195, 295-316. 
Marchis, F., et al., 2008b. Main belt binary asteroidal systems with circular mutual orbits. Icarus 196, 97-118.

Margot, J.L., Brown, M.E., 2003. A low-density M-type asteroid in the main belt. Science 300, 1939-1942.

Margot, J.L., et al., 2002. Binary asteroids in the near-Earth object population. Science 296, $1445-1448$.

Margot, J.L., et al., 2006. Hermes as an exceptional case among binary near-Earth asteroids. IAU Gen. Assem. 236.

Margot, J.L., et al., 2008. Detailed characterization of Asteroid (35107) 1991 VH. Bull. Am. Astron. Soc. 40, (Abstract \#25.06).

Marzari, F., Davis, D., Vanzani, V., 1995. Collisional evolution of asteroid families. Icarus 113, 168-187.

Marzari, F., Farinella, P., Davis, D.R., 1999. Origin, aging, and death of asteroid families. Icarus $142,63-77$.

McMahon, J., Scheeres, D.J., 2010a. Secular orbit variation due to solar radiation effects: A detailed model for BYORP. Celest. Mech. Dynam. Astron. 106, 261-300.

McMahon, J., Scheeres, D.J., 2010b. Detailed prediction for the BYORP effect on binary near-Earth Asteroid (66391) 1999 KW4 and implications for the binary population. Icarus 209, 494-509.

Merline, W.J., et al., 2000. Satellites of minor planets. IAU Circ. 7503, 3.

Merline, W.J., et al., 2001. S/2001 (617) 1. IAU Circ. 7741, 2.

Merline, W.J., Weidenschilling, S.J., Durda, D.D., Margot, J.L., Pravec, P., Storrs, A.D., 2002. Asteroids do have satellites. In: Bottke, W.F., Cellino, A., Paolicchi, P., Binzel, R.P. (Eds.), Asteroids III. Univ. of Arizona Press, Tucson, pp. 289-312.

Michałowski, T., et al., 2004. Eclipsing binary Asteroid 90 Antiope. Astron. Astroph. 423, $1159-1168$.

Mueller, M., et al., 2010. Eclipsing binary Trojan Asteroid Patroclus: Thermal inertia from Spitzer observations. Icarus 205, 505-515.

Murray, C.D., Dermott, S.F., 1999. Solar System Dynamics. Cambridge Univ. Press, Cambridge.

Nesvorný, D., Morbidelli, A., Vokrouhlický, D., Bottke, W.F., Brož, M., 2002a. The Flora family: A case of the dynamically dispersed collisional swarm? Icarus 157, 155-172.

Nesvorný, D., Bottke, W.F., Dones, L., Levison, H.F., 2002b. The recent breakup of an asteroid in the main-belt region. Nature 417, 720-722. 
Nesvorný, D., Bottke, W.F., Levison, H.F., Dones, L., 2003. Recent origin of the Solar System dust bands. Astroph. J. 591, 486-497.

Nesvorný, D., Jedicke, R., Whiteley, R.J., Ivezić, Ž., 2005. Evidence for asteroid space weathering from the Sloan Digital Sky Survey. Icarus 173, 132-152.

Nesvorný, D., Youdin, A.N., Richardson, D.C., 2010. Formation of Kuiper Belt binaries by gravitational collapse. Astron. J. 140, 785-793.

Nolan, M.C., Howell, E.S., Miranda, G., 2004. Radar images of binary Asteroid 2003 YT1. Bull. Am. Astron. Soc. 36, (Abstract \#28.08).

Nolan, M.C., Howell, E.S., Becker, T.M., Magri, C., Giorgini, J.D., Margot, J.L., 2008. Arecibo radar observations of 2001 SN263: A near-Earth triple asteroid system. Bull. Am. Astron. Soc. 40, (Abstract \#25.04).

Noll, K.S., Grundy, W.M., Chiang, E.I., Margot, J.L., Kern, S.D., 2008. Binaries in the Kuiper belt. In: Barucci, M.A., Boehnhardt, H., Cruikshank, D.P., Morbidelli, A. (Eds.), The Solar System Beyond Neptune. Univ. of Arizona Press, Tucson, pp. 345-363.

Ostro, S.J., et al., 2006. Radar imaging of binary near-Earth Asteroid (66391) 1999 KW4. Science 314, 1276-1280.

Peale, S.J., Lissauer, J.J., 1989. Rotation of Halley's comet. Icarus 79, 396-430.

Pravec, P., Harris, A.W., 2007. Binary asteroid population 1. Angular momentum content. Icarus 190, 250-259.

Pravec, P., et al., 2006. Photometric survey of binary near-Earth asteroids. Icarus 181, 63-93.

Richardson, D.C., Walsh, K.J., 2006. Binary minor planets. Annu. Rev. Earth Planet. Sci. 34, $47-81$.

Richardson, D.C., Bottke, W.F., Love, S.G., 1998. Tidal distortion and disruption of Earth-crossing asteroids. Icarus 134, 47-76.

Richardson, D.C., Leinhardt, Z.M., Melosh, H.J., Bottke, W.F., Asphaug, E., 2002. Gravitational aggregates: Evidence and evolution. In: Bottke, W.F., Cellino, A., Paollichi, P., Binzel, R.P. (Eds.), Asteroids III. Univ. of Arizona Press, Tucson, pp. 501-515.

Rojo, P., Margot, J.L., 2011. Mass and density of the B-type Asteroid (702) Alauda. Astroph. J. 727, 69-73.

Rubincam, D.P., 2000. Radiative spin-up and spin-down of small asteroids. Icarus 148, 2-11.

Scheeres, D.J., 2006. Relative equilibria for general gravity fields in the sphere-restricted full 2-body problem. Celest. Mech. Dynam. Astron. 94, 317-349.

Scheeres, D.J., 2007a. Rotational fission of contact binary asteroids. Icarus 189, 370-385. 
Scheeres, D.J., 2007b. The dynamics of NEO binary asteroids. In: Milani, A., Valsecchi, G.B., Vokrouhlický, D. (Eds.), Near Earth Objects, our Celestial Neighbors: Opportunity and Risk. Cambridge Univ. Press, Cambridge, pp. 177-190.

Scheeres, D.J., 2009. Stability of the planar full 2-body problem. Cel. Mech. Dynam. Astron. 104, $103-128$.

Scheeres, D.J., et al., 2006. Dynamical configuration of binary near-Earth Asteroid (66391) 1999 KW4. Science 314, 1280-1283.

Taylor, P.A., 2009. Preferential production of contact binary asteroids with components of similar mass via angular momentum loss. AAS/Div. Dynam. Astron. Meet. 40, (Abstract \#12.03).

Taylor, P.A., Margot, J.L., 2010. Tidal evolution of close binary asteroid systems. Cel. Mech. Dynam. Astron. 108, 315-338.

Taylor, P.A., et al., 2007. Spin rate of Asteroid (54509) 2000 PH5 increasing due to the YORP effect. Science 316, 274-277.

Taylor, P.A., et al., 2008. The shape, mutual orbit, and tidal evolution of binary near-Earth Asteroid 2004 DC. LPI Contrib. 1405, (Abstract \#8322).

Vokrouhlický, D., Čapek, D., 2002. YORP-induced long-term evolution of the spin state of small asteroids and meteoroids: Rubincam's approximation. Icarus 159, 449-467.

Vokrouhlický, D., et al., 2006. Yarkovsky footprints in the Eos family. Icarus 182, 92-117.

Walsh, K.J., Richardson, D.C., 2006. Binary near-Earth asteroid formation: Rubble pile model of tidal disruptions. Icarus 180, 201-216.

Walsh, K.J., Richardson, D.C., Michel, P., 2008. Rotational breakup as the origin of small binary asteroids. Nature 454, 188-191.

Weidenschilling, S.J., Paolicchi, P., Zappalà, V., 1989. Do asteroids have satellites? In: Binzel, R.P., Gehrels, T., Matthews, M.S. (Eds.), Asteroids II. Univ. of Arizona Press, Tucson, pp. 643-660.

Wisdom, J., Peale, S.J., Mignard, F., 1984. The chaotic rotation of Hyperion. Icarus 58, 137-152.

Yoder, C.F., 1982. Tidal rigidity of Phobos. Icarus 49, 327-346.

Zappalà, V., Bendjoya, P., Cellino, A., Farinella, P., Froeschle, C., 1995. Asteroid families: Search of a 12,487-asteroid sample using two different clustering techniques. Icarus 116, 291-314.

This manuscript was prepared with the AAS LATEX macros v5.0. 